\title{
Full Abstraction for the Quantum Lambda-Calculus
}

\author{
PIERRE CLAIRAMBAULT, Univ Lyon, EnsL, UCBL, CNRS, LIP, LYON, France \\ MARC DE VISME, Univ Lyon, EnsL, UCBL, CNRS, LIP, LYON, France
}

Quantum programming languages permit a hardware independent, high-level description of quantum algorithms. In particular, the quantum $\lambda$-calculus is a higher-order language with quantum primitives, mixing quantum data and classical control. Giving satisfactory denotational semantics to the quantum $\lambda$-calculus is a challenging problem that has attracted significant interest. In the past few years, both static (the quantum relational model) and dynamic (quantum game semantics) denotational models were given, with matching computational adequacy results. However, no model was known to be fully abstract.

Our first contribution is a full abstraction result for the games model of the quantum $\lambda$-calculus. Full abstraction holds with respect to an observational quotient of strategies, obtained by summing valuations of all states matching a given observable. Our proof method for full abstraction extends a technique recently introduced to prove full abstraction for probabilistic coherence spaces with respect to probabilistic PCF.

Our second contribution is an interpretation-preserving functor from quantum games to the quantum relational model, extending a long line of work on connecting static and dynamic denotational models. From this, it follows that the quantum relational model is fully abstract as well.

Altogether, this gives a complete denotational landscape for the semantics of the quantum $\lambda$-calculus, with static and dynamic models related by a clean functorial correspondence, and both fully abstract.

CCS Concepts: • Theory of computation $\rightarrow$ Denotational semantics; • Computer systems organization $\rightarrow$ Quantum computing.

Additional Key Words and Phrases: Quantum Programming, Full Abstraction, Game Semantics

ACM Reference Format:

Pierre Clairambault and Marc de Visme. 2020. Full Abstraction for the Quantum Lambda-Calculus. Proc. ACM Program. Lang. 4, POPL, Article 63 (January 2020), 28 pages. https://doi.org/10.1145/3371131

\section{INTRODUCTION}

Quantum computation promises to have a huge impact in computing. Algorithms like Shor's [Shor 1997] or Grover's [Grover 1996] challenge our traditional view of algorithmics and complexity, and applications exploiting quantum features in cryptography [Gisin et al. 2002] are already deployed. The field is moving fast, with large companies investing massively in the race for quantum hardware.

To accompany this trend, researchers have developed programming languages for quantum computing. The quantum $\lambda$-calculus [Selinger and Valiron 2006] is a paradigmatic such language, marrying quantum computation with classical control. Finding denotational semantics for the quantum $\lambda$-calculus has attracted a lot of attention, and over the years, models were given for various fragments [Delbecque 2011; Hasuo and Hoshino 2017; Malherbe 2013; Malherbe et al. 2013; Selinger and Valiron 2008]. An adequate denotational semantics for the full language was finally achieved six years ago by Pagani, Selinger and Valiron [Pagani et al. 2014] and presented at POPL'14

Authors' addresses: Pierre Clairambault, Univ Lyon, EnsL, UCBL, CNRS, LIP, LYON, F-69364 LYON Cedex 07, France, Pierre.Clairambault@ens-lyon.fr; Marc de Visme, Univ Lyon, EnsL, UCBL, CNRS, LIP, LYON, F-69364 LYON Cedex 07 France, Marc.de-Visme@ens-lyon.fr.

This work is licensed under a Creative Commons Attribution 4.0 International License.

(C) 2020 Copyright held by the owner/author(s).

2475-1421/2020/1-ART63

https://doi.org/10.1145/3371131

Proc. ACM Program. Lang., Vol. 4, No. POPL, Article 63. Publication date: January 2020 
Their model enriches the relational model [Ehrhard 2012] with annotations from the category CPM of completely positive maps, a natural mathematical framework for (first-order) quantum computing - in this paper, we shall refer to their model as the quantum relational model. Finally, Clairambault, de Visme and Winskel presented another adequate model of the full language [Clairambault et al. 2019], enriching the game semantics of [Castellan et al. 2019] with annotations from CPM.

In denotational semantics, the gold standard for the match between a language and its semantics is full abstraction [Milner 1977], meaning that the equivalence induced by the model captures exactly observational equivalence: two terms have the same denotation if and only if they cannot be distinguished within the syntax. Over the decades, fully abstract models have been given for a myriad of languages - game semantics [Abramsky et al. 2000; Hyland and Ong 2000] contributing its fair share. For quantum programming, Selinger and Valiron have proved that (a linear version of) the quantum relational model is fully abstract for the linear fragment of the quantum $\lambda$-calculus [Selinger and Valiron 2008]. But for the full language, full abstraction remains open.

Until recently, there were few examples of full abstraction results for languages with quantitative features such as probabilities or quantum effects. Indeed, most tools traditionally used to construct fully abstract models struggle with quantitative aspects. Most full abstraction results are achieved by showing that a significant fragment of the model - representative of its dynamic behaviour but finite in some way - is definable in the syntax: for instance, almost all full abstraction results in game semantics proceed in this way. For a quantitative language this seems hard to do: the mathematical space used is larger, with non-trivial interactions between the control flow and quantitative aspects. The task of capturing precisely the image of the interpretation seems daunting.

Fortunately, a new methodology to prove full abstraction for quantitative languages emerged recently. The same year as the first adequate denotational model for the quantum $\lambda$-calculus, also in POPL'14, Ehrhard, Tasson and Pagani presented a proof that probabilistic coherence spaces are fully abstract for probabilistic PCF [Ehrhard et al. 2014]. Their method is striking by its originality: they showed that from terms with distinct interpretations one could extract - by feeding them to test terms weighted by formal parameters - characteristic power series, then exploit regularity properties of analytic functions to separate these series, hence the terms. These two POPL'14 papers raise the question: could a similar method achieve full abstraction for the quantum $\lambda$-calculus?

Contributions. In this paper, we give a positive answer to this question.

Our first contribution is to prove that the games model of [Clairambault et al. 2019] is fully abstract for the quantum $\lambda$-calculus. Of course, full abstraction does not hold up to the very intensional equivalence on strategies considered in [Clairambault et al. 2019]. Instead, we prove it with respect to an observational quotient, obtained by summing the valuations of all states of a strategy leading to a given observable outcome. Our proof of full abstraction is strongly inspired by [Ehrhard et al. 2014], however the construction is heavily impacted by the presence of quantum effects. To extract characteristic power series compositionally, we must extend the model so that states of configurations carry formal polynomials over CPM maps, rather than merely CPM maps. Furthermore, given two terms whose interpretation yield distinct CPM maps on a given observable state, we must first find adequate quantum measurements to be performed by test terms before we are reduced to the probabilistic case. Then, we can conclude as in [Ehrhard et al. 2014].

Our second contribution is to connect the two adequate models of the quantum $\lambda$-calculus, the quantum relational model of [Pagani et al. 2014] and the game semantics model of [Clairambault et al. 2019]; via an interpretation-preserving functor from games to quantum relations. From our interpretation-preserving functor, it follows that the quantum relational model is also fully abstract.

Related work on the relational collapse. This quantum relational collapse extends an active line of research on linking dynamic models such as games with static ones such as the relational model. 
Very early on, researchers have investigated the relationship between game semantics and relational semantics, noting in particular that the natural time-forgetting operation from games to relations is not functorial ${ }^{1}$ [Baillot et al. 1997]. This has to do with the dynamic aspect of games which makes them sensitive to deadlocks in compositions, unlike relational semantics. However, for deterministic innocent strategies - which capture semantically pure programs [Hyland and Ong 2000], it was proved by Melliès [Melliès 2005, 2006] (in asynchronous games) and Boudes [Boudes 2009] that no deadlocks can arise during composition, making the collapse functorial.

These collapse results require innocence - or at least a substitute ensuring that composition is deadlock-free. But beyond the sequential deterministic case, there was for a long time no adequate notion of innocence [Harmer and McCusker 1999]. This changed only a few years ago, with two notions of non-deterministic innocent strategies (using concurrent games [Castellan et al. 2014] and sheaves [Tsukada and Ong 2015]). These two models depart from traditional game semantics in ways that are technically very different, but conceptually similar: they both record more intensional behavioural information. This change of perspective recently allowed a quantitative extension of the relational collapse [Castellan et al. 2018] for a probabilistic language, using concurrent games.

Concurrent games are a family of game semantics initiated in [Abramsky and Melliès 1999], with intense activity in the past decade prompted by a new non-deterministic generalization based on event structures [Rideau and Winskel 2011]. Building on notions from concurrency theory, they are a natural fit for the semantics of concurrent programs [Castellan and Clairambault 2016; Castellan and Yoshida 2019]. It is perhaps more surprising that their adoption has a strong impact even when studying sequential programs such as the quantum $\lambda$-calculus: they offer a fine-grained causal presentation of the behaviour of programs that contrasts with the temporal presentation of traditional games models. This has far-reaching consequences. For the present paper, both our collapse theorem and the congruence of the observational quotient required for full abstraction rely on a visibility condition, a substitute for innocence ensuring a deadlock-free composition visibility bans certain impure causal patterns, leveraging the expressiveness of concurrent games.

Thus, our constructions rely heavily on the fact that the model of [Clairambault et al. 2019] was developed within concurrent games. Our collapse theorem follows in the footsteps of the probabilistic collapse [Castellan et al. 2018], which we generalize to the quantum case.

Outline. In Section 2 we introduce the quantum $\lambda$-calculus and give some preliminaries on the mathematics of quantum computation. In Section 3 we describe our variant of the games model of [Clairambault et al. 2019], differing notably in that we allow annotations by polynomials over CPM, and in that we adopt an exhaustivity discipline due to Melliès to ban arbitrary weakening. In Section 4 we define the observational quotient, and prove the associated convergence and congruence properties. Finally, in Section 5, we prove full abstraction for games, then give the functorial collapse to the quantum relation model for which we deduce full abstraction.

\section{QUANTUM $\lambda$-CALCULUS AND PRELIMINARIES}

We start by introducing the quantum $\lambda$-calculus [Pagani et al. 2014]. To allow us later on to build the test terms weighted by formal parameters, we will extend the language with those.

\subsection{The Parametrized Quantum $\lambda$-Calculus}

\subsubsection{Syntax and Types. The types of the quantum $\lambda$-calculus are given by:}

$$
A, B::=\text { qbit }|1| A \otimes B|A \oplus B| A^{\ell}|A \multimap B| !(A \multimap B)
$$

\footnotetext{
${ }^{1}$ In fact, there is a functor from deterministic sequential games to relations [Hyland and Schalk 1999], but it is not monoidal so it does not preserve the interpretation. It may be refined into a monoidal functor [Calderon and McCusker 2010], but with respect to a new monoidal structure incompatible with the usual relational interpretation of the $\lambda$-calculus.
} 


$$
\begin{aligned}
& \frac{(A \text { linear })}{! \Gamma, x: A \vdash x: A} \quad \frac{! \Gamma \vdash v: A \multimap B}{! \Gamma \vdash v: !(A \multimap B)} \quad \frac{\Gamma, x: A \vdash t: B}{! \Gamma \vdash \text { skip }: 1} \quad \frac{}{\Gamma \vdash \lambda x^{A} \cdot t: A \multimap B} \\
& \frac{! \Gamma, \Delta \vdash t: A \multimap B \quad ! \Gamma, \Omega \vdash u: A}{! \Gamma, \Delta, \Omega \vdash t u: B} \quad \frac{! \Gamma, \Delta \vdash t: 1 \quad ! \Gamma, \Omega \vdash u: A}{! \Gamma, \Delta, \Omega \vdash t ; u: A} \quad \frac{! \Gamma, \Delta \vdash t: A \quad ! \Gamma, \Omega \vdash u: B}{! \Gamma, \Delta, \Omega \vdash t \otimes u: A \otimes B} \\
& \frac{! \Gamma, \Delta \vdash t: A \otimes B \quad ! \Gamma, \Omega, x: A, y: B \vdash u: C}{! \Gamma, \Delta, \Omega \vdash \operatorname{let} x^{A} \otimes y^{B}=t \text { in } u: C} \quad \frac{! \Gamma, \Delta \vdash t: A_{1} \oplus A_{2} \quad ! \Gamma, \Omega, x: A_{i} \vdash u_{i}: C}{! \Gamma, \Delta, \Omega \vdash \operatorname{match} t \text { with }\left(x^{A_{1}}: u_{1} \mid x^{A_{2}}: u_{2}\right): C} \quad \frac{\Gamma \vdash t: A}{\Gamma \vdash \operatorname{in}_{l}(t): A \oplus B} \\
& \frac{\Gamma \vdash u: B}{\Gamma \vdash \operatorname{in}_{r}(t): A \oplus B} \quad \frac{\Gamma \vdash t: 1 \oplus\left(A \otimes A^{\ell}\right)}{\Gamma \vdash t: A^{\ell}} \quad \frac{! \Gamma, f: !(A \multimap B), x: A \vdash t: B \quad \Delta, ! \Gamma, f: !(A \multimap B) \vdash u: C}{\Delta, ! \Gamma \vdash \operatorname{letrec} f^{A-\circ B} x^{A}=t \operatorname{in} u: C} \\
& \overline{\Gamma \vdash \text { split : } A^{\ell} \multimap 1 \oplus\left(A \otimes A^{\ell}\right)} \quad \overline{! \Gamma \vdash \text { meas : qbit } \multimap \text { bit }} \quad \overline{! \Gamma \vdash \text { new : bit } \multimap \text { qbit }} \quad \frac{U \text { unitary of arity } n}{! \Gamma \vdash U: \text { qbit }^{\otimes n} \multimap \text { qbit }^{\otimes n}}
\end{aligned}
$$

Fig. 1. Typing rules for the quantum $\lambda$-calculus

The type qbit represents qubits, the quantum equivalent of bits and atomic pieces of quantum data. We also have a unit type 1 along with tensors (whose inhabitants are pairs), sums and finite lists (with, as a particular case, the type of integers nat $=1^{\ell}$ ). Classical bits are defined as syntactic sugar via bit $=1 \oplus 1$. There are two function types: $(A \multimap B)$ for functions that may be used any number of times, and $A \multimap B$ for functions that have to be used exactly once. As in [Pagani et al. $2014]$, ! is restricted to function types. This forbids the unrealistic type !qbit of replicable qubits. However, ! $(1 \multimap$ qbit $)$ makes perfect sense: its elements are functions called arbitrarily many times, which at each call generate a new independent qubit. Types of the form ! $(A \multimap B)$ are non-linear, while all the others are linear. We now introduce the grammar of terms:

$$
\begin{aligned}
t, u::= & x\left|\lambda x^{A} . t\right| t u|\operatorname{skip}| t ; u|t \otimes u| \text { let } x^{A} \otimes y^{B}=t \operatorname{in} u\left|\operatorname{in}_{l} t\right| \text { in }_{r} t \\
& \mid \text { match } t \text { with }\left(x^{A}: u_{1} \mid y^{B}: u_{2}\right)|\operatorname{split}| \text { letrec } f^{A-B} x^{A}=t \text { in } u \mid \text { new } \mid \text { meas } \mid U
\end{aligned}
$$

Apart from the last three constructors, this describes a simply-typed $\lambda$-calculus with unit, tensor, sums, lists, and recursive definitions. Hopefully any ambiguities concerning the syntax should be cleared up by the typing rules. Constructors for lists may be defined as syntactic sugar, by [] = $\operatorname{in}_{l}$ skip and $t:: u=\operatorname{in}_{r}(t \otimes u)$. Likewise, we set $t \mathrm{t}=\operatorname{in}_{l}$ skip, ff $=\operatorname{in}_{r}$ skip, and if $M$ then $N_{1}$ else $N_{2}$ may be defined as match $M$ with $\left(x^{1}: N_{1} \mid y^{1}: N_{2}\right)$. We sometimes use additional syntactic sugar, provided it is unambiguous how it should be defined within the quantum $\lambda$-calculus.

The last three constructors are quantum primitives. The first, new : bit $\multimap$ qbit, prepares a new qbit based on a given bit. The second, meas : qbit $\multimap$ bit, performs a measurement. Finally, $U:$ qbit $^{\otimes n} \multimap$ qbit $^{\otimes n}$ (where qbit ${ }^{\otimes n}$ is the iterated tensor qbit $\otimes \ldots \otimes$ qbit) stands for any unitary map of arity $n$ - the language includes a primitive for every unitary. The mathematical meaning of these will be reviewed in Section 2.2, where we recall some quantum preliminaries.

Before we go on to typing, we give the grammar of values.

$$
v, w::=x\left|\lambda x^{A} . t\right| v \otimes w\left|\operatorname{in}_{l} v\right| \operatorname{in}_{r} v \mid \text { skip } \mid \text { split } \mid \text { meas } \mid \text { new } \mid U
$$

Typing judgements have the form $\Gamma \vdash t: A$ with $\Gamma$ a context, i.e. a list of declarations of distinct variables $x_{1}: A_{1}, \ldots, x_{n}: A_{n}$. We say that $\Gamma$ is non-linear iff it has the form $x_{1}: ! A_{1}, \ldots x_{n}: ! A_{n}$; we may then write ! $\Gamma$ to emphasize this. Most typing rules are displayed in Figure 1 . To these we add an exchange rule allowing us to permute variable declarations in contexts - having an explicit exchange helps in writing a clean definition of the denotational semantics.

In this paper we will rely heavily on the adequate model of the quantum $\lambda$-calculus introduced in [Clairambault et al. 2019]. With respect to that paper, our version of the quantum $\lambda$-calculus differs in that its !-free fragment is linear rather than affine. We make this choice merely to ease the 
link with the model of [Pagani et al. 2014], which relies on linearity. Note that the adequacy result of [Clairambault et al. 2019] also applies to the present variant: each program typable with a linear discipline is obviously typable with an affine discipline. We omit the (call-by-value) operational semantics, which we will only link to through the adequacy result of [Clairambault et al. 2019].

For example programs in the quantum $\lambda$-calculus, the reader is directed to [Pagani et al. 2014].

2.1.2 Parametrized Extension. Drawing inspiration from [Ehrhard et al. 2014], the proof of full abstraction will rely on an extension of the language. Typing judgments have the form $\Gamma \vdash \mathcal{p} M: A$, where $\mathcal{P}$ is a set of formal parameters taken from a fixed countable set disjoint from other syntactic constructs $-\mathcal{P}$ is the set of parameters that may appear in $M$. We add the new typing rule

$$
\frac{\Gamma \vdash \mathcal{P} M: A}{\Gamma \vdash \mathcal{P} \mathrm{X} \cdot M: A}(\mathrm{X} \in \mathcal{P})
$$

for each $\mathrm{X} \in \mathcal{P}$. Other typing rules leave the annotation by $\mathcal{P}$ unchanged.

Intuitively, parameters range over $[0,1]$. Given $\alpha \in[0,1]$ and $\vdash M: A$, there is $\Gamma \vdash \alpha \cdot M: A$ a term acting like $M$ with probability $\alpha$ and otherwise diverging (in [Ehrhard et al. 2014], formal parameters could only be instantiated with rationals as their language only allows as primitive probabilistic choice weighted with rational coefficients - in contrast, our language contains a constant for arbitrary unitary transforms, and $\alpha \cdot M$ can be defined for any $\alpha \in[0,1]$. This distinction does not change much as far as the full abstraction argument is concerned.). For $M_{1}, \ldots, M_{n}$ homogeneously typed and $\alpha_{1}, \ldots, \alpha_{n}$ with $\sum_{1 \leq i \leq n} \alpha_{i} \leq 1$, we write $\sum_{1 \leq i \leq n} \alpha_{i} \cdot M_{i}$ for the weighted sub-probabilistic sum, which is definable in the language. If $\Gamma \vdash \mathcal{P} M: A$ and $\rho \in[0,1]^{\mathcal{P}}$, we write $\Gamma \vdash M[\rho]: A$ for $M$ with every formal parameter $\mathrm{X}$ replaced with $\rho(\mathrm{X}) \in[0,1]$.

In the sequel, we will not need to extend the operational semantics of the quantum $\lambda$-calculus in the presence of formal parameters. We shall however extend game semantics [Clairambault et al. 2019] with those, in a way that is compatible with substitution of formal parameters with scalars.

\subsection{Quantum Preliminaries}

Pure quantum states, as stored in a quantum store when executing programs, are usually represented as normalized vectors in a finite-dimensional Hilbert space - in this paper all Hilbert spaces will be finite-dimensional, so we will drop the "finite-dimensional" qualifier and leave it implicit. For example, qubits are represented as normalized vectors in the Hilbert space $\mathbb{C}^{2}:$ it is customary to write those $\alpha|0\rangle+\beta|1\rangle \in \mathbb{C}^{2}$, with $(|0\rangle,|1\rangle)$ the canonical basis of $\mathbb{C}^{2}$ as a $\mathbb{C}$-vector space.

As quantum measurement is probabilistic, the evaluation of quantum programs naturally yields probability (sub)distributions of pure states, which, rather than simply vectors in Hilbert spaces, will be certain linear maps operating on Hilbert spaces - one then speaks of mixed quantum states. Such mixed states play a central role for the denotational semantics of the quantum $\lambda$-calculus; accordingly we describe below some of the associated mathematical structure.

2.2.1 Hilbert Spaces. Let Hilb be the category of Hilbert spaces and linear maps, which is wellknown to be symmetric monoidal; write $\otimes$ for its tensor product and $I$ for its unit, which is simply the space $\mathbb{C}$ of complex numbers. It is further compact closed: any Hilbert space $H$ has a dual $H^{*}=\operatorname{Hilb}(H, I)$, with a unit $\eta_{H}: I \rightarrow H^{*} \otimes H$ and a co-unit $\epsilon_{H}: H \otimes H^{*} \rightarrow I$. Via this compact closed structure Hilb admits a partial trace (to form a traced monoidal category [Joyal et al. 1996]). Given $f: H \otimes L \rightarrow K \otimes L$ in Hilb, its partial trace is a map $\operatorname{Tr}_{L}(f): H \rightarrow K$, obtained as in Figure 2. If $f: L \rightarrow L$, its (complete) trace is $\operatorname{tr}(f)=\operatorname{Tr}_{I}(I \otimes f): I \rightarrow I$ so a scalar factor, matching the usual

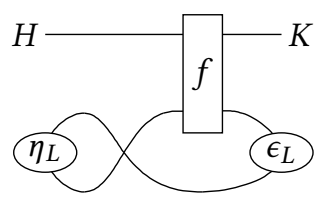

Fig. 2. Partial trace trace of the matrix of $f$. Indeed, $\operatorname{Hilb}(L, L)$ is isomorphic to $L^{*} \otimes L$ whose vectors we can think of as 
matrices. A unitary map is $f: H \rightarrow K$ in Hilb which is invertible with inverse $f^{-1}=f^{\dagger}: K \rightarrow H$, given by its conjugate transpose.

2.2.2 Positive Operators. An operator is a linear map with the same domain and codomain. An operator $f: H \rightarrow H$ in Hilb is positive if it is hermitian, i.e $f=f^{\dagger}$, and its eigenvalues are non-negative real numbers. Write $\operatorname{Op}(H)$, and $\operatorname{Pos}(H)$, for the set of operators, respectively positive operators, on $H$. We equip $\mathrm{Op}(H)$ with an order, the Löwner order (see e.g. [Selinger 2004]), by $f \leq_{L} g$ iff $g-f \in \operatorname{Pos}(H)$. Those $\rho \in \operatorname{Pos}(H)$ for which $\operatorname{tr}(\rho) \leq 1$ are the subdensity operators.

Subdensity operators represent mixed quantum states, quantum states closed under probability (sub)distributions. For instance, subdensity operators on $\mathbb{C}^{2}$ represent mixed quantum states on one qubit: a pure qubit $\alpha|0\rangle+\beta|1\rangle$ appears as $\left(\begin{array}{cc}|\alpha|^{2} & \alpha \bar{\beta} \\ \bar{\alpha} \beta & |\beta|^{2}\end{array}\right)$. Here $|\alpha|^{2}$ and $|\beta|^{2}$ are reals and sum to 1 , one may think of $|\alpha|^{2}$ as the probability of measuring ff, of $|\beta|^{2}$ as that of measuring $\mathrm{tt}$, and the other coefficients as required to express the behaviour of the state under unitary transforms. More generally, a pure state expressed as a map $f: I \rightarrow H$ in Hilb yields $\widehat{f}=f f^{\dagger} \in \operatorname{Pos}(H)$ a density operator that can be also represented as a density matrix. So, subdensity operators can represent pure states - but unlike those, they are also stable under convex (sub-probabilistic) sums.

2.2.3 Completely Positive Maps. Whereas positive operators can represent mixed states, completely positive maps express transformations that take mixed states to mixed states. The category CPM again has Hilbert spaces as objects, but now a map $f \in \mathbf{C P M}(H, K)$ is a linear map $f: H^{*} \otimes H \rightarrow K^{*} \otimes K$ in Hilb such that its correspondent $\bar{f}: H^{*} \otimes K \rightarrow H^{*} \otimes K$, got by compact closure (Figure 3), is positive. The 1-1 correspondence $f \mapsto \bar{f}$ between completely positive maps and positive operators is known as the Choi-famiolkowski isomorphism.

CPM inherits from Hilb its compact closed structure. It is helpful conceptually and technically to regard $f \in \mathbf{C P M}(H, K)$ in $\mathbf{C P M}$ as taking

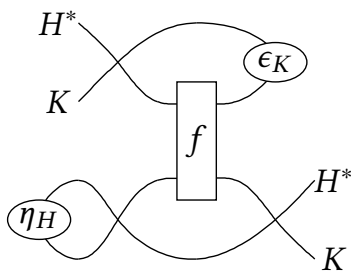

Fig. 3. Construction of $\bar{f}$ operators on $H$ to operators on $K$, so as $f: \mathrm{Op}(H) \rightarrow \mathrm{Op}(K)$ in Hilb. A linear map $f: \mathrm{Op}(H) \rightarrow$ $\mathrm{Op}(K)$ is positive if it takes positive operators to positive operators. Those $f: \mathrm{Op}(H) \rightarrow \mathrm{Op}(K)$ arising from completely positive maps are those for which $f \otimes \mathrm{id}_{L}$ is positive for any $\operatorname{id}_{L}: \mathrm{Op}(L) \rightarrow$ $\mathrm{Op}(L)$. If a completely positive map $f$ further satisfies $\operatorname{tr}(f(A)) \leq \operatorname{tr}(A)$ it is called a superoperator, which capture physically realisable operations.

We can describe a map in CPM, regarded as a map between operators, as mapping matrices to matrices linearly. For instance the measurement of a value 0 or 1 of a qubit in $\mathbb{C}^{2}$ is described, respectively, by the two superoperators meas $_{0}$, meas $_{1} \in \mathrm{CPM}\left(\mathbb{C}^{2}, I\right)$ where

$$
\operatorname{meas}_{0}:\left(\begin{array}{ll}
a & b \\
c & d
\end{array}\right) \mapsto a \text { and } \text { meas }_{1}:\left(\begin{array}{ll}
a & b \\
c & d
\end{array}\right) \mapsto d
$$

Symmetrically, the two superoperators new , new $_{1} \in \operatorname{CPM}\left(I, \mathbb{C}^{2}\right)$ represent initialization:

$$
\text { new }_{0}: a \mapsto\left(\begin{array}{cc}
a & 0 \\
0 & 0
\end{array}\right) \quad \text { and } \quad \text { new }_{1}: d \mapsto\left(\begin{array}{ll}
0 & 0 \\
0 & d
\end{array}\right)
$$

Finally, for $f: H \rightarrow K$ a unitary, the superoperator $\widehat{f} \in \mathbf{C P M}(H, K)$ takes $g \in \mathbf{O p}(H)$ to $f g f^{\dagger}$.

2.2.4 Parametrized Completely Positive Maps. To match the extension of the language with formal parameters, we will rely on quantum annotations themselves extended with formal parameters again, this methodology is the same as in [Ehrhard et al. 2014] in the probabilistic case.

For $H$ a Hilbert space and $\mathcal{P}=\left\{\mathrm{X}_{1}, \ldots, \mathrm{X}_{n}\right\}$ a finite set of parameters, we write $\operatorname{Pos}(H)[\mathcal{P}]$ for the set of multivariate polynomials with coefficients in $\operatorname{Pos}(H)$. More precisely, a monomial is a finite 
multiset of parameters in $\mathcal{P}$ - write $\mathcal{M}(\mathcal{P})$ for this set. A multivariate polynomial is a function $\mathcal{M}(\mathcal{P}) \rightarrow \operatorname{Pos}(H)$ associating to each monomial a coefficient in $\operatorname{Pos}(H)$, and which has finite support: there is a finite number of monomials with a non-zero coefficient. We denote multivariate polynomials as formal sums, in expressions such as (with $\alpha, \beta, \gamma \in \operatorname{Pos}(H)$ and $\mathrm{X}, \mathrm{Y}, \mathrm{Z} \in \mathcal{P}$ )

$$
\alpha+\beta \mathrm{X}^{2} \mathrm{Y}+\gamma \mathrm{XY}+\delta \mathrm{Z} \in \operatorname{Pos}(H)[\mathcal{P}],
$$

where e.g. $\alpha$ is associated with the trivial monomial and $\beta$ with the monomial $\mathrm{X}^{2} \mathrm{Y}$, omitting monomials with null coefficient. Multivariate polynomials support a sum, defined pointwise.

Likewise, given two Hilbert spaces $H, K$, the set $\mathrm{CPM}[\mathcal{P}](H, K)$ comprises multivariate polynomials with coefficients in $\mathbf{C P M}(H, K)$. Given $P=\sum_{i \in I} \alpha_{i} m_{i} \in \mathbf{C P M}[\mathcal{P}](H, K)$ and $Q=\sum_{j \in J} \beta_{j} m_{j}^{\prime} \in$ $\mathrm{CPM}[\mathcal{P}](K, L)$, their composition is defined through polynomial multiplication, as in:

$$
Q \circ P=\sum_{(i, j) \in I \times J}\left(\beta_{j} \circ \alpha_{i}\right)\left(m_{i} m_{j}^{\prime}\right) \in \operatorname{CPM}[\mathcal{P}](H, L)
$$

where the product of monomials $m_{i} m_{j}^{\prime}$ is the sum of multisets. This definition follows the expansion of composition of polynomials, relying implicitely on linearity of composition in CPM. This makes $\mathrm{CPM}[\mathcal{P}]$ a category with objects Hilbert spaces, morphisms from $H$ to $K$ the set $\mathrm{CPM}[\mathcal{P}](H, K)$, composition as above and identity on $H$ the polynomial with only non-zero coefficient $\mathrm{id}_{H}^{\mathrm{CPM}} \in$ $\mathbf{C P M}(H, H)$, attached to the trivial monomial. The tensor $P_{1} \otimes P_{2} \in \mathbf{C P M}[\mathcal{P}]\left(H_{1} \otimes H_{2}, K_{1} \otimes K_{2}\right)$ of $P_{1} \in \mathbf{C P M}[\mathcal{P}]\left(H_{1}, K_{1}\right)$ and $P_{2} \in \mathbf{C P M}[\mathcal{P}]\left(H_{2}, K_{2}\right)$ is defined analogously, relying on the product of monomials and the monoidal product of CPM. Just as CPM, СPM $[\mathcal{P}]$ is compact closed.

The formal parameters in $\mathrm{CPM}[\mathcal{P}]$ reflect those in our extended language; and similarly they can be substituted for values in $[0,1]$. If $m$ is a monomial (on parameters $\mathcal{P}$ ) and $\rho \in[0,1]^{\mathcal{P}}$, define the substitution $m[\rho]=\Pi_{\mathrm{X} \in \mathcal{P}} \rho(X)^{m(\mathrm{X})} \in[0,1]$. If $P=\sum_{i \in I} \alpha_{i} m_{i}$ is in $\operatorname{Pos}(H)[\mathcal{P}]$, define

$$
P[\rho]=\sum_{i \in I}\left(m_{i}[\rho]\right) \alpha_{i} \in \operatorname{Pos}(H)
$$

and likewise for $P \in \mathbf{C P M}[\mathcal{P}](H, K)$. Substitution defines a strict compact closed functor $-[\rho]$ : $\mathrm{CPM}[\mathcal{P}] \rightarrow \mathrm{CPM}$ : it commutes with all operations involved in the compact closed structure.

\section{PARAMETRIZED QUANTUM GAME SEMANTICS}

Though our games model is mostly the same as [Clairambault et al. 2019], it differs in two respects.

Firstly, for the connection with the quantum relational model of Section 5.3 to work, we need our games model to enforce linearity strictly for the !-free fragment. To that end we import the payoff mechanism introduced by Melliès in [Melliès 2005] to achieve full completeness for linear logic.

Secondly, to match formal parameters in the syntax, our quantum annotations will no longer be in $\mathrm{CPM}$, but in $\mathrm{CPM}[\mathcal{P}]$ for some finite set $\mathcal{P}$. Though this difference might look significant, the construction of [Clairambault et al. 2019] unfolds in much the same way in CPM and CPM[P] as it relies mostly on the compact closed structure of the category of annotations.

By lack of space, our exposition is unfortunately rather succinct. The reader is directed to [Clairambault et al. 2019] for a more slow-paced presentation of the model.

\subsection{Linear Exhaustive Games}

In this subsection, we present linear exhaustive games. In most aspects, this is the same category of games and strategies as introduced in [Rideau and Winskel 2011] and detailed in [Castellan et al. 2017]. To this, we add a mechanism to express which strategies are strictly linear, in the sense that they consume all available linear resources. Though our terminology evokes [Murawski and Ong 2003], their method is too tied to sequential games. Instead we adapt the constructions of Melliès 


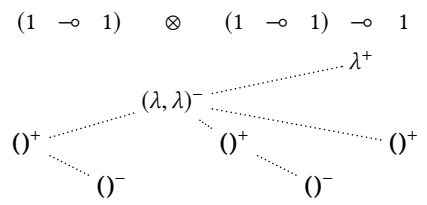

Fig. 4. The ev. str. for $(1 \multimap 1) \otimes(1 \multimap 1) \multimap 1$.

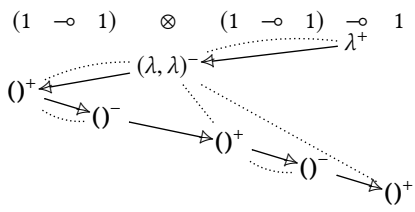

Fig. 5. A strategy on $(1 \multimap 1) \otimes(1 \multimap 1) \multimap 1$

[Melliès 2005; Melliès and Tabareau 2010], which also bears similarity with the technique based on realizability developed in [Dal Lago and Laurent 2008] for a similar purpose.

\subsubsection{Games and Exhaustive Strategies. Our games and strategies are certain event structures.}

Definition 3.1. An event structure (es) is $\left(E, \leq_{E}, \mathrm{Con}_{E}\right)$ where $E$ is a set of events partially ordered by $\leq_{E}$ the causal dependency relation, and $\mathrm{Con}_{E}$ is a nonempty consistency relation consisting of finite subsets of $E$. These are subject to the following additional axioms:

$$
\begin{aligned}
& {[e]_{E}=\operatorname{def}\left\{e^{\prime} \mid e^{\prime} \leq_{E} e\right\} \text { is finite for all } e \in E,} \\
& \{e\} \in \operatorname{Con}_{E} \text { for all } e \in E, \\
& Y \subseteq X \in \operatorname{Con}_{E} \text { implies } Y \in \operatorname{Con}_{E}, \text { and } \\
& X \in \operatorname{Con}_{E} \& e \leq_{E} e^{\prime} \in X \text { implies } X \cup\{e\} \in \operatorname{Con}_{E} .
\end{aligned}
$$

All event structures are assumed countable, with an injection from events to natural numbers left implicit. An event structure with polarities (esp) also has a polarity function $\operatorname{pol}_{E}: E \rightarrow\{-,+\}$.

We often drop $E$ in $\leq_{E}, \operatorname{Con}_{E},[e]_{E}$ when clear from the context. When introducing an event in the presence of polarities, we might annotate it to set its polarity, as in $e^{+}, e^{-}$.

The relation $e^{\prime} \leq_{E} e$ expresses that $e$ causally depends on the earlier occurrence of event $e^{\prime}$. That a finite subset of events is consistent conveys that its events can occur together by some stage in the evolution of the process. Event structures come with a notion of state: a (finite) configuration is a finite $x \subseteq E$ which is consistent, i.e. $x \in \mathrm{Con}_{E}$, and down-closed, i.e. for all $e \in x$, for all $e^{\prime} \leq_{E} e$ we have $e^{\prime} \in x$ as well. We write $\mathscr{C}(E)$ for the set of all configurations of $E$. We also write $e \rightarrow_{E} e^{\prime}$, called immediate causal dependency iff $e<_{E} e^{\prime}$ with no event strictly in between.

In our interpretation, a game presents all computational actions available in a call-by-value evaluation on a certain type, along with their (in)compatibilities and their causal dependencies.

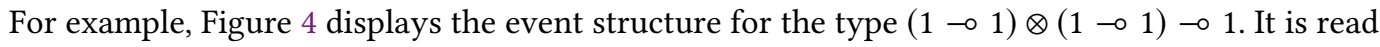
from top to bottom, with dotted lines representing immediate causal dependency - when drawing games and strategies for/on a type, we always attempt to draw events under the corresponding type component. Under call-by-value evaluation, the first available action is $\lambda^{+}$, corresponding to the program under consideration evaluating to a $\lambda$-abstraction. The evaluation environment may then feed a value, which consists in two $\lambda$-abstractions $(\lambda, \lambda)^{-}$(as the argument is a tensor of functions). The program may then return (with ()$^{+}$on the right), or feed an argument to either or both of the two functions (with moves ()$^{+}$on the left) which may then return (with moves ()$^{-}$).

A game is an esp with further components guaranteeing exhaustivity.

Definition 3.2. A game is $\left(A, \kappa_{A}\right)$ where (1) $A$ is an esp which is race-free, i.e. if $x, x \cup\left\{a_{1}^{+}\right\}, x \cup$ $\left\{a_{2}^{-}\right\} \in \mathscr{C}(A)$, then $x \cup\left\{a_{1}, a_{2}\right\} \in \mathscr{C}(A) ;(2) \kappa_{A}: \mathscr{C}(A) \rightarrow\{-1,0,+1\}$ is a payoff function.

The payoff function $\kappa_{A}$ partitions $\mathscr{C}(A)$ in three sets. We write $\mathcal{E}_{A}=\left\{x \in \mathscr{C}(A) \mid \kappa_{A}(x)=0\right\}$, and we think of those as exhaustive configurations, representing completed computations matching the resource constraints. For instance, in the game arising as the interpretation of $(1 \multimap 1) \otimes(1 \multimap 1) \multimap 1$, 
only the full configuration is exhaustive (we will see in Section 5.3 that exhaustive configurations match points of the web in the relational model). But strategies can also reach non-exhaustive configurations, because (1) game semantics display the non-exhaustive intermediate stages leading eventually to a final exhaustive state; and (2) computation might terminate on a non-exhaustive configuration if Opponent does not behave exhaustively, e.g. performs weakenings. Configurations $x \in \mathscr{C}(A)$ such that $\kappa_{A}(x)=+1$ are those non-exhaustive configurations where however the responsibility of non-exhaustiveness can be assigned to Opponent. Likewise, in configurations $x \in \mathscr{C}(A)$ such that $\kappa_{A}(x)=-1$, the responsibility of non-exhaustiveness is assigned to Player.

For strategies, we first recall the notion from [Castellan et al. 2017; Rideau and Winskel 2011].

Definition 3.3. A strategy on game $A$ is an es $S$, with a labelling function $\sigma: S \rightarrow A$, which is:

(1) Rule-abiding. For any $x \in \mathscr{C}(S), \sigma x \in \mathscr{C}(A)$,

(2) Local injectivity. If $s, s^{\prime} \in x \in \mathscr{C}(S)$ and $\sigma s=\sigma s^{\prime}$, then $s=s^{\prime}$.

(3) Receptive. If $x \in \mathscr{C}(S)$ and $\sigma x$ extends with negative $a^{-} \in A$, i.e. $a \notin \sigma x$ and $\sigma x \cup\{a\} \in \mathscr{C}(A)$, then there is a unique $s \in S$ such that $\sigma s=a$ and $x \cup\{s\} \in \mathscr{C}(S)$.

(4) Courteous. If $s_{1} \rightarrow_{S} s_{2}$, then either $\sigma s_{1} \rightarrow_{A} \sigma s_{2}$, or $\operatorname{pol}_{A}\left(\sigma s_{1}\right)=-\operatorname{and~}_{\operatorname{pol}_{A}}\left(\sigma s_{2}\right)=+$.

A strategy specifies which events of the game it is prepared to play, and under which additional causal constraints. To deal with non-determinism it is convenient to separate the set of events of the strategy from that of the game, because the same event in the game may occur several times in the strategy under incompatible non-deterministic branches. In a strategy $\sigma: S \rightarrow A$, we think of $S$ as the strategy and $\sigma$ as the labelling map annotating each event of $S$ with the corresponding event in the game. Conditions (1) and (2) amount to $\sigma$ being a map of event structures, and conditions (3) and (4) ensure that a strategy must acknowledge all Opponent moves, and may only add further causal constraints from Opponent moves to Player moves. Figure 5 represents a strategy (that of $\lambda f^{1-1} \otimes g^{1-01} \cdot g$ ( $f$ skip), with a slight abuse of notations) on the game of Figure 4. When representing strategies we display the event structure $S$, but with an event $s$ labelled as $\sigma s \in A$. We show immediate causal links in $S$ as $\rightarrow$ and in $A$ as dotted lines.

If $\sigma: S \rightarrow A$ is a strategy, any $s \in S$ inherits a polarity from $A$ : by $\operatorname{pol}(s)$ we mean $\operatorname{pol}_{A}(\sigma s)$. A configuration $x \in \mathscr{C}(S)$ is +-covered if all its maximal events (for $\leq_{S}$ ) have positive polarity. Intuitively, +-covered configurations correspond to "stopping states" of the strategy.

Definition 3.4. A strategy $\sigma: S \rightarrow A$ is exhaustive iff $\forall x \in \mathscr{C}(S)$ +-covered, we have $\kappa_{A}(\sigma x) \geq 0$.

In other words, " $\sigma x$ is exhaustive, or it is Opponent's fault". If $A$ is a game, then its dual $A^{\perp}$ is obtained by reversing polarities and setting $\kappa_{A^{\perp}}=-\kappa_{A}$, all other components unchanged.

Exhaustivity resembles winning conditions [Clairambault et al. 2012], in which one assigns to each configuration a status: winning (+1) or losing (-1), but not neutral (0). For $\sigma: S \rightarrow A$, in settings with winning conditions [Clairambault et al. 2012] we think of some $\tau: T \rightarrow A^{\perp}$ as a counter-strategy - it is then impossible to have both $\sigma$ and $\tau$ be winning. In contrast here we can have both $\sigma$ and $\tau$ exhaustive, in which case if $x \in \mathscr{C}(S)$ and $y \in \mathscr{C}(T)$ are +-covered such that $\sigma x=\tau y$, then $\sigma x \in \mathcal{E}_{A}$. Closed interactions between exhaustive strategies must be exhaustive.

3.1.2 $\star$-Autonomous Structure. We explore the compositional structure of games and strategies.

If $A$ and $B$ are esp, their simple parallel composition, written $A \| B$, has events the tagged disjoint union $\{1\} \times A \cup\{2\} \times B$ and other components inherited - we write any $x \in \mathscr{C}(A \| B)$ as $x_{A} \| x_{B}$ accordingly. For games, this yields two distinct operations $A \otimes B$ (notation chosen to avoid 
collision with $\otimes)$ and $A \not \supset B$. For those, we first define operations $\otimes$ and $\not \supset$ on $\{-1,0,+1\}$ as

\begin{tabular}{c|ccc}
$\otimes$ & -1 & 0 & +1 \\
\hline-1 & -1 & -1 & -1 \\
0 & -1 & 0 & +1 \\
+1 & -1 & +1 & +1
\end{tabular}

\begin{tabular}{c|ccc}
$x$ & -1 & 0 & +1 \\
\hline-1 & -1 & -1 & +1 \\
0 & -1 & 0 & +1 \\
+1 & +1 & +1 & +1
\end{tabular}

and then set $\kappa_{A \otimes B}\left(x_{A} \| x_{B}\right)=\kappa_{A}\left(x_{A}\right) \otimes \kappa_{B}\left(x_{B}\right)$ and $\kappa_{A}$ × $_{B}\left(x_{A} \| x_{B}\right)=\kappa_{A}\left(x_{A}\right)$ > $\kappa_{B}\left(x_{B}\right)$.

In particular, $\mathcal{E}_{A \bowtie B}=\mathcal{E}_{A^{\ngtr} \mathrm{B}}=\left\{x_{A} \| x_{B} \mid x_{A} \in \mathcal{E}_{A} \& x_{B} \in \mathcal{E}_{B}\right\}$, in bijection with $\mathcal{E}_{A} \times \mathcal{E}_{B}$. The operations $\otimes$ and $\varnothing$ are dual, i.e. $(A \otimes B)^{\perp}=A^{\perp} \not \varnothing B^{\perp}$. We write $\varnothing$ for the game with no events and $\kappa_{\varnothing}(\emptyset)=0-$ it is a unit for both $\otimes$ and $\not \supset$. An exhaustive strategy from $A$ to $B$ is an exhaustive strategy $\sigma: S \rightarrow A^{\perp} \not 2 B$; occasionally written $\sigma: A \rightarrow B$ keeping $S$ anonymous.

From $\sigma: A \rightarrow B$ and $\tau: B \rightarrow C$ we wish to define $\tau \odot \sigma: A \rightarrow C$ resulting from their interaction this relies on the following definition. Fix exhaustive strategies $\sigma: S \rightarrow A^{\perp} \| B$ and $\tau: T \rightarrow B^{\perp} \| C$.

Definition 3.5. Configurations $x_{S} \in \mathscr{C}(S)$ and $x_{T} \in \mathscr{C}(T)$ are causally compatible iff (1) $\sigma x_{S}=$ $x_{A} \| x_{B}$ and $\tau x_{T}=x_{B} \| x_{C}$, and (2) the induced composite bijection $\varphi$

$$
x_{S}\left\|x_{C} \stackrel{\sigma \| x_{C}}{\cong} x_{A}\right\| x_{B}\left\|x_{C} \stackrel{x_{A} \| \tau^{-1}}{\cong} x_{A}\right\| x_{T}
$$

is secured, i.e. the relation $(c, d) \triangleleft\left(c^{\prime}, d^{\prime}\right) \Leftrightarrow\left(c \leq_{S \| C} c^{\prime} \vee d \leq_{A \| T} d^{\prime}\right)$ on (the graph of $) \varphi$ is acyclic.

A causally compatible $\left(x_{S}, x_{T}\right)$ is minimal iff it is minimal amongst causally compatible pairs with the same projections on $A$ and $B$, ordered by the product of the inclusions.

Causally compatible pairs are the expected states of the interaction between $\sigma$ and $\tau$ - the matching condition expresses that configurations agree on the interface, and securedness that they do not impose incompatible causal constraints; in other words they synchronize without deadlock

To define composition, we rely on the following proposition.

Proposition 3.6. There is a strategy $\tau \odot \sigma: T \odot S \rightarrow A^{\perp} \not \gamma C$, unique up to isomorphism, such that there is an order-isomorphism between minimal causally compatible pairs $\left(x_{S}, x_{T}\right)$ and configurations $z \in \mathscr{C}(T \odot S)$ (we write $z=x_{T} \odot x_{S}$ to emphasize this correspondence), and such that writing $\sigma x_{S}=x_{A} \| x_{B}$ and $\tau x_{T}=x_{B} \| x_{C}$, we then have $(\tau \odot \sigma)\left(x_{T} \odot x_{S}\right)=x_{A} \| x_{C}$.

Moreover, $\tau \odot \sigma: A \rightarrow C$ is exhaustive.

Here, isomorphism between strategies $\sigma: S \rightarrow A$ and $\sigma^{\prime}: S^{\prime} \rightarrow A$ means a bijection $\varphi: S \cong S^{\prime}$ preserving and reflecting all structure, making the obvious triangle commute.

We now define the identities, the copycat strategies. For $x, y$ configurations of a game $A$ we write $x \subseteq^{-} y$ iff $x \subseteq y$ and $\operatorname{pol}(y \backslash x) \subseteq\{-\}$; and symmetrically for $x \subseteq^{+} y$.

Proposition 3.7. For a game $A$ there is a unique exhaustive $\boldsymbol{c}_{A}: \mathbb{C}_{A} \rightarrow A^{\perp} \not>A$ with events $\mathbb{C}_{A}=A^{\perp} \| A, \mathbf{c}_{A}$ the identity, and configurations all $x_{A} \| y_{A}$ such that $x_{A} \supseteq^{+} x_{A} \cap y_{A} \subseteq^{-} y_{A}$.

Copycat is neutral for composition, up to iso. If $\sigma_{1}: S_{1} \rightarrow A_{1}^{\perp} \not \gamma B_{1}$ and $\sigma_{2}: S_{2} \rightarrow A_{2}^{\perp} \not 8 B_{2}$, their tensor $\sigma_{1} \otimes \sigma_{2}: S_{1} \| S_{2} \rightarrow\left(A_{1} \otimes A_{2}\right)^{\perp} \not \gamma\left(B_{1} \otimes B_{2}\right)$ is defined as the obvious relabeling, and likewise for $\sigma_{1} \gamma \sigma_{2}$ - both functorial up to iso. Finally, these data form a linearly distributive category with negation [Cockett and Seely 1997], which is equivalent to a $\star$-autonomous category.

COROLlary 3.8. Games and exhaustive strategies form a $a$-autonomous category.

\subsection{Parametrized Quantum Annotations}

The enrichment with quantum annotations closely follows [Clairambault et al. 2019], with the distinction that valuations are in $\mathrm{CPM}[\mathcal{P}]$ for some set $\mathcal{P}$ of formal parameters, rather than in CPM. The model aims to reflect the principle of classical control, quantum data: classical control is embodied by a strategy, over which sits annotations representing quantum data. 


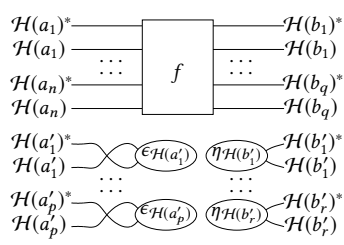

Fig. 6. Expansion $\left\lceil y_{A}, y_{B}(f)\right.$

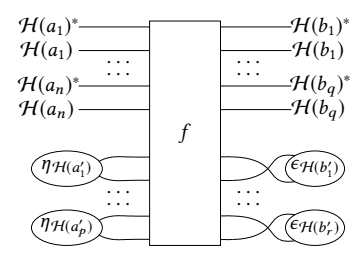

Fig. 7. Reduction $\bigvee_{x_{A}, x_{B}}(f)$

3.2.1 Quantum Games and Strategies. Firstly, in our games, each event will contribute a Hilbert space. If the event comes from a type component with no quantum data (such as bit or 1), this Hilbert space will be trivial (i.e. the one-dimensional Hilbert space $I=\mathbb{C}$ ). However, if the event comes from qbit $^{\otimes n}$, then the associated Hilbert space will have dimension $2^{n}$.

Definition 3.9. A quantum game $\left(A, \kappa_{A}, \mathcal{H}_{A}\right)$ consists of a game, together with $\mathcal{H}_{A}: A \rightarrow$ Hilb associating, to any event in $A$, a Hilbert space.

Each finite set $x \subseteq A$ carries a Hilbert space $\mathcal{H}_{A}(x)=\bigotimes_{a \in x} \mathcal{H}_{A}(a)-$ in particular $\mathcal{H}_{A}(\emptyset)=I$. Our earlier constructions on games are easily extended to quantum games, by stating $\mathcal{H}_{A^{\perp}}(a)=$ $\mathcal{H}_{A}(a)^{*}$ (the dual space), and $\mathcal{H}_{A \bowtie B}=\mathcal{H}_{A{ }^{x} B}$ associates $(1, a)$ to $\mathcal{H}_{A}(a)$ and $(2, b)$ to $\mathcal{H}_{B}(b)$, so $e$.g $\mathcal{H}_{A \otimes B}\left(x_{A} \| x_{B}\right)=\mathcal{H}_{A}\left(x_{A}\right) \otimes \mathcal{H}_{B}\left(x_{B}\right)$. For example, the type qbit will be interpreted as the quantum game with only one (positive) event, written $\mathbf{q}^{+}$, with $\kappa(\emptyset)=-1$ and $\kappa\left(\left\{\mathbf{q}^{+}\right\}\right)=0$; and $\mathcal{H}\left(\mathbf{q}^{+}\right)=\mathbb{C}^{2}$

We now define quantum strategies. As they must form a category, we directly define what is a strategy from one game to another. If $\sigma: S \rightarrow A^{\perp} \gamma \mathrm{B}$ is a plain strategy for quantum games $A$ and $B$, then each $x \in \mathscr{C}(S)$ projects as $\sigma x=x_{A} \| x_{B}$ for $x_{A} \in \mathscr{C}(A)$ and $x_{B} \in \mathscr{C}(B)$. The configuration $x \in \mathscr{C}(S)$ expresses the current state in the control flow, i.e. the classical part of computation. To this, we must add quantum data. For that, we observe that $x_{A}$ and $x_{B}$ induce Hilbert spaces $\mathcal{H}_{A}\left(x_{A}\right)$ and $\mathcal{H}_{B}\left(x_{B}\right)$; so we can adjoin quantum data as a valuation $Q_{\sigma}(x) \in \operatorname{CPM}\left(\mathcal{H}_{A}\left(x_{A}\right), \mathcal{H}_{B}\left(x_{B}\right)\right)$.

However, quantum data is not completely decorrelated from classical control - a condition is used to tame how much the quantum valuation can change locally throughout computation. This condition, though strictly speaking unnecessary to obtain a model, is what lets us keep coefficients finite in the basic model construction, which will play a crucial role in the full abstraction proof. The condition appears below (the notations $\bigvee$ and $\bigvee$ will be introduced after the definition).

Definition 3.10. For $A$ and $B$ quantum games, a quantum strategy from $A$ to $B$ is an exhaustive strategy $\sigma: S \rightarrow A^{\perp} \not 2 B$ with a valuation $Q^{\rightarrow}:(x \in \mathscr{C}(S)) \rightarrow \mathrm{CPM}[\mathcal{P}]\left(\mathcal{H}_{A}\left(x_{A}\right), \mathcal{H}_{B}\left(x_{B}\right)\right)$ satisfying:

- Normalised: $Q^{\rightarrow}(\emptyset)=\operatorname{id}_{I} \in \operatorname{CPM}[\mathcal{P}](I, I)$,

- Oblivious: If $x \subseteq^{-} y$ with $\sigma y=y_{A} \| y_{B}$, then $Q^{\rightarrow}(y)=\gamma^{y_{A}, y_{B}}\left(Q^{\rightarrow}(x)\right)$,

- Monotone: For $y \subseteq^{+} x_{1}, \cdots, x_{n}, d_{Q^{\rightarrow}}\left[y ; x_{1}, \ldots, x_{n}\right] \in \operatorname{CPM}[\mathcal{P}]\left(y_{A}, y_{B}\right)$, where

$$
d_{Q \rightarrow}\left[y ; x_{1}, \ldots, x_{n}\right]=Q^{\rightarrow}(y)-\sum_{\emptyset \neq I \subseteq\{1, \ldots, n\}}(-1)^{|I|+1} \bigvee_{y_{A}, y_{B}}\left(Q^{\rightarrow}\left(x_{I}\right)\right),
$$

with $Q^{\rightarrow}\left(x_{I}\right)=Q^{\rightarrow}\left(\bigcup_{i \in I} x_{i}\right)$ when the union is a configuration and the zero map otherwise.

The difficulty in constraining how the quantum valuations can change locally, is that the ambiant Hilbert space is not invariant: it grows as new moves are played, opening up new qubits. This impacts axioms oblivious and monotone: for the former, the condition may be thought of as asking that if $x \subseteq^{-} y$ in $\mathscr{C}(S)$, the valuation on $y$ is that of $x$ extended to $y$ by tracing out the new spaces opponed up by Opponent moves - this is the purpose of the operation $\lceil$. For the latter, different $x_{I}$ s correspond to different augmentations of the spaces $\mathcal{H}_{A}\left(y_{A}\right)$ and $\mathcal{H}_{B}\left(y_{B}\right)$, as the positive moves 
may have opponed new qubits as well. These new spaces are traced out in the sum, bringing each term down to $\mathrm{CPM}[\mathcal{P}]\left(\mathcal{H}_{A}\left(y_{A}\right), \mathcal{H}_{B}\left(y_{B}\right)\right)$ - this is the purpose of the operation $\bigvee$.

At first ignoring parameters in $\mathcal{P}$, if $x_{A}\left\|x_{B} \subseteq y_{A}\right\| y_{B} \in \mathscr{C}\left(A^{\perp} \| B\right)$, the expansion

$$
\nwarrow^{y_{A}, y_{B}}(f) \in \operatorname{CPM}[\mathcal{P}]\left(\mathcal{H}_{A}\left(y_{A}\right), \mathcal{H}_{B}\left(y_{B}\right)\right)
$$

of $f \in \operatorname{CPM}\left(\mathcal{H}_{A}\left(x_{A}\right), \mathcal{H}_{B}\left(x_{B}\right)\right)$ to $y_{A}, y_{B}$ is defined as in Figure 6 (with $x_{A}=\left\{a_{1}, \ldots, a_{n}\right\}, x_{B}=$ $\left\{b_{1}, \ldots b_{q}\right\}, y_{A} \backslash x_{A}=\left\{a_{1}^{\prime}, \ldots, a_{p}^{\prime}\right\}$ and $\left.y_{B} \backslash x_{B}=\left\{b_{1}^{\prime}, \ldots, b_{r}^{\prime}\right\}\right)$ using the compact closed structure of Hilb. This is then extended to $\mathbf{C P M}[\mathcal{P}]$ monomial per monomial. For $f \in \operatorname{CPM}[\mathcal{P}]\left(\mathcal{H}_{A}\left(y_{A}\right), \mathcal{H}_{B}\left(y_{B}\right)\right)$, its reduction $\bigvee_{x_{A}, x_{B}}(f) \in \mathrm{CPM}[\mathcal{P}]\left(\mathcal{H}_{A}\left(x_{A}\right), \mathcal{H}_{B}\left(x_{B}\right)\right)$ is defined likewise (Figure 7).

The sum in the definition of $d_{Q \rightarrow}\left[y ; x_{1}, \ldots, x_{n}\right]$ is performed monomial per monomial, so this amounts to the condition in [Clairambault et al. 2019] applied separately for each monomial. This adapts and extends the inclusion-exclusion principle used for probabilistic strategies [Winskel 2015], the reader is directed to [Clairambault et al. 2019] for more details and intuitions. Using the compact closed structure of $\mathrm{CPM}$, any $\mathrm{Q}^{\rightarrow}(x) \in \mathrm{CPM}\left(\mathcal{H}\left(x_{A}\right), \mathcal{H}\left(x_{B}\right)\right)$ can be reorganised as a map in $\operatorname{CPM}\left(\mathcal{H}(\sigma x)^{-}, \mathcal{H}(\sigma x)^{+}\right)$, from the Hilbert space corresponding to the negative events to those for the positive events. It is then proved in [Clairambault et al. 2019] that it is in fact a superoperator.

\subsubsection{Categorical Structure. We extend the structure of Section 3.1.2 to quantum games.}

Proposition 3.11. Let $\sigma: S \rightarrow A^{\perp} \not 8 B$ and $\tau: T \rightarrow B^{\perp} \not 8 C$ be two quantum strategies. Setting

$$
Q_{\tau \odot \sigma}^{\overrightarrow{0}}\left(x_{T} \odot x_{S}\right)=Q_{\tau}\left(x_{T}\right) \circ Q_{\sigma}\left(x_{S}\right) \in \operatorname{CPM}[\mathcal{P}]\left(\mathcal{H}_{A}\left(x_{A}\right), \mathcal{H}_{C}\left(x_{C}\right)\right)
$$

for every $x_{T} \odot x_{S} \in \mathscr{C}(T \odot S)$ makes $\tau \odot \sigma$ a quantum strategy.

So the valuation of composed states amounts to composition in CPM $[\mathcal{P}]$. Likewise, the tensor of $\sigma_{1}: S_{1} \rightarrow A_{1}^{\perp} \not 8 B_{1}$ and $\sigma_{2}: S_{2} \rightarrow A_{2}^{\perp} \not 8 B_{2}, \sigma_{1} \otimes \sigma_{2}$ and $\sigma_{1} \not 8 \sigma_{2}$ are made into quantum strategies by $Q_{\sigma_{1} \boxplus \sigma_{2}}\left(x_{1} \| x_{2}\right)={\overrightarrow{Q_{\sigma_{1}} \gamma \sigma_{2}}}_{\overrightarrow{2}}\left(x_{1} \| x_{2}\right)=Q_{\sigma_{1}}\left(x_{1}\right) \otimes Q_{\sigma_{2}}\left(x_{2}\right)$, using the monoidal structure of CPM $[\mathcal{P}]$.

Finally, we need to equip copycat $\boldsymbol{c}_{A}: \mathbb{C}_{A} \rightarrow A^{\perp} \| A$ with a quantum valuation. As composition of quantum strategies relies on composition in $\mathrm{CPM}[\mathcal{P}]$, we expect the quantum valuation of copycat to rely on the identities in $\mathrm{CPM}[\mathcal{P}]$, i.e. those in CPM. Indeed, for balanced configurations of the form $x \| x \in \mathscr{C}\left(\mathbb{C}_{A}\right)$, we set $Q_{\mathbf{c}_{A}}(x \| x)=\operatorname{id}_{\mathcal{H}(x)}: \mathcal{H}(x) \stackrel{\mathrm{CPM}}{\rightarrow} \mathcal{H}(x)$. In general, configurations of copycat are $x \| y$ where $x \supseteq^{+} x \cap y \subseteq^{-} y$; i.e. a balanced $x \cap y \| x \cap y \in \mathscr{C}\left(\mathbb{C}_{A}\right)$ with a negative extension to $x \| y$. By obliviousness, the definition is forced to be

$$
{Q_{\mathbf{c}_{A}}}(x \| y)=\bigvee^{x, y}\left(\operatorname{id}_{\mathcal{H}(x \cap y)}\right): \mathcal{H}(x) \stackrel{\text { PPM }}{\rightarrow} \mathcal{H}(y) .
$$

COROLlARY 3.12. Quantum games and quantum strategies forms a

\subsection{Extension with Symmetry}

The above can serve as canvas to interpret the !-free fragment of the quantum $\lambda$-calculus. For the full language we must deal with replication and recursion; which as usual in concurrent games requires us to extend games with symmetry. In essence, adding symmetry consists in replicating the developments above in the more expressive event structures with symmetry [Winskel 2007].

Definition 3.13. A symmetry on an event structure $E$ is a set $\cong_{E}$ comprising bijections $\theta: x \cong y$ where $x, y \in \mathscr{C}(E)$ are configurations (we write $\theta: x \cong_{E} y$ if $\theta \in \cong_{E}$ ) satisfying:

- Groupoid. The set $\cong_{E}$ comprises identities and is closed under inverse and composition.

- Restriction. For any $\theta: x \cong_{E} y$ and $x^{\prime} \subseteq x$ such that $x^{\prime} \in \mathscr{C}(E)$, there exists a (necessarily unique) $\theta^{\prime} \subseteq \theta$ such that $\theta^{\prime}: x^{\prime} \cong_{E} y^{\prime}$;

- Expansion. For $\theta: x \cong_{E} y$ and $x \subseteq x^{\prime} \in \mathscr{C}(E)$, there exists some $\theta \subseteq \theta^{\prime}$ s.t. $\theta^{\prime}: x^{\prime} \cong_{E} y^{\prime}$. 
We regard $\cong_{E}$ as a proof-relevant equivalence relation - we will write simply $x \cong_{E} y$ for the corresponding equivalence relation. The last two conditions amount to $\cong_{E}$ being a history-preserving bisimulation. We refer to elements of $\cong_{E}$ as symmetries. It follows from "restriction" that symmetries are order-isomorphisms (with configurations ordered by $\leq_{E}$ ). Two events $e_{1}, e_{2} \in E$ are symmetric (written $\left.e_{1} \cong_{E} e_{2}\right)$ iff $\left(e_{1}, e_{2}\right) \in \theta \in \cong_{E}$ for some $\theta$; or equivalently if $\left[e_{1}\right] \cong_{E}\left[e_{2}\right]$.

Symmetry, when added to games, is the concurrent games counterpart of the equivalence relation on plays in AJM games [Abramsky et al. 2000]. It helps us relate strategies which behave in the same way, but only up to symmetry; which is crucial as the laws of ! in Section 3.4 only hold up to symmetry. However, it is hard to build a notion of "behaving in the same way up to symmetry" that is also preserved under composition. The solution of [Castellan et al. 2015, 2019] relies on the introduction of the two subsymmetries $\cong_{A}^{-}$and $\cong_{A}^{+}-$intuitively, $\cong_{A}^{-}$comprises those symmetries where only Opponent has changed their copy indices, and dually for $\cong_{A}^{+}$. To the conditions of [Castellan et al. 2019] we add a new requirement that any configuration has a canonical representative, which we need for the observational quotient.

Definition 3.14. A -game comprises $\left(A, \kappa_{A}, \mathcal{H}_{A}, \cong_{A}, \cong_{A}^{+}, \cong_{A}^{-}\right)$where $(1)\left(A, \kappa_{A}, \mathcal{H}_{A}\right)$ is a quantum game; and $(2) \cong_{A}, \cong_{A}^{+}$and $\cong_{A}^{-}$are three symmetries s.t. $\cong_{A}^{+}, \cong_{A}^{-} \subseteq_{A}$, satisfying the conditions of thin concurrent games [Castellan et al. 2019]. To these we add that for all $x_{A} \in \mathscr{C}(A)$, there is some symmetric $x_{A} \cong_{A} y_{A}$ such that $y_{A}$ is canonical, in the sense that any symmetry $\theta: y_{A} \cong_{A} y_{A}$ decomposes (necessarily uniquely) as $\theta^{+} \circ \theta^{-}$, where $\theta^{+}: y_{A} \cong_{A}^{+} y_{A}$ and $\theta^{-}: y_{A} \cong_{A}^{-} y_{A}$.

Finally, we require that $\kappa_{A}$ is stable under $\cong_{A}$, and that if $a \cong_{A} a^{\prime}$, then $\mathcal{H}_{A}(a)=\mathcal{H}_{A}\left(a^{\prime}\right)$.

Any $\theta: x \cong_{A} y$ induces a unitary between $\mathcal{H}(x)$ and $\mathcal{H}(y)$ obtained by the action of $\theta$ on the tensors $\mathcal{H}(x)=\bigotimes_{a \in x} \mathcal{H}(a)$ and $\mathcal{H}(y)=\bigotimes_{a \in y} \mathcal{H}(a)$; we write it $\mathcal{H}(\theta): \mathcal{H}(x) \cong \mathcal{H}(y)$. Earlier constructions on games extend: $\cong_{A^{\perp}}=\cong_{A}, \cong_{A^{\perp}}^{+}=\cong_{A}^{-}$and $\cong_{A^{\perp}}^{-}=\cong_{A}^{+}$. Likewise, $\cong_{A \bowtie B}=\cong_{A^{\ngtr} \mathrm{B}}$ comprises $\theta_{A}\left\|\theta_{B}: x_{A}\right\| x_{B} \cong y_{A} \| y_{B}$ such that $\theta_{A}: x_{A} \cong_{A} y_{A}$ and $\theta_{B}: x_{B} \cong_{B} y_{B}$.

Definition 3.15. A -strategy on $A$ is a quantum strategy $\sigma: S \rightarrow A$ with $\cong_{S}$ on $S$, subject to

- Symmetry-preservation. If $\theta: x \cong_{S} y$, then $\sigma \theta=\left\{\left(\sigma s_{1}, \sigma s_{2}\right) \mid\left(s_{1}, s_{2}\right) \in \theta\right\}: \sigma x \cong_{A} \sigma y$;

- Strong-receptivity. If $\theta: x \cong_{S} y$, if $\sigma \theta \cup\left\{\left(a_{1}^{-}, a_{2}^{-}\right)\right\}: x \cup\left\{a_{1}\right\} \cong_{A} y \cup\left\{a_{2}\right\}$, then $\theta \cup\left\{\left(s_{1}, s_{2}\right)\right\}$ : $x \cup\left\{s_{1}\right\} \cong_{S} y \cup\left\{s_{2}\right\}$ where $s_{1}, s_{2}$ such that $\sigma s_{1}=a_{1}, \sigma s_{2}=a_{2}$ come from receptivity;

- Thin. If $x \in \mathscr{C}(S)$, if $\operatorname{id}_{x} \subseteq^{+} \theta \in \cong_{S}$, then $\theta=\operatorname{id}_{y}$ for some $y \in \mathscr{C}(S)$;

from [Castellan et al. 2019]. Additionally, we impose compatibility of quantum valuations with symmetry: for any $\theta: x \cong_{S} y$, writing $\sigma \theta=\theta_{A} \| \theta_{B}$ with $\theta_{A}: x_{A} \cong_{A} y_{A}$ and $\theta_{B}: x_{B} \cong_{B} y_{B}$,

$$
Q_{\sigma}(y)=\overline{\mathcal{H}\left(\theta_{B}\right)} \circ Q_{\sigma}^{\vec{\sigma}}(x) \circ{\overline{\mathcal{H}\left(\theta_{A}\right)}}^{-1} .
$$

We formalize what it means to "behave the same up to symmetry". Two $\sim$-strategies $\sigma: S \rightarrow$ $A^{\perp} \not 8 \mathrm{~B}$ and $\sigma^{\prime}: S^{\prime} \rightarrow A^{\perp} \not \gamma B$ are weakly isomorphic iff there is a bijection $\varphi: S \cong S^{\prime}$ reflecting and preserving all structure (including symmetry and quantum valuations), and such that for all $x \in \mathscr{C}(S)$, we have $\left\{\left(\sigma s, \sigma^{\prime}(\varphi s)\right) \mid s \in x\right\} \in \cong_{A^{\perp} x_{B}}$. It is one of the mains results of [Castellan et al. 2019] that weak isomorphism is preserved under composition and the other constructions.

Corollary 3.16. For each finite set $\mathcal{P}$ of parameters, $\sim$-games and $\sim$-strategies up to weak isomorphism form a $a$-autonomous category $\sim-\mathrm{QCG}[\mathcal{P}]$.

From now on, by strategy we always mean -strategy.

\subsection{Interpretation}

We will interpret the quantum $\lambda$-calculus with parameters in $\mathcal{P}$ into $\sim-\mathrm{QCG}[\mathcal{P}]$. However the interpretation does not target $\sim-\mathrm{QCG}[\mathcal{P}]$ directly, but relies on a derived structure fit for the 
interpretation of call-by-value. We keep the description of this construction as brief as possible; it is the same as in [Clairambault et al. 2019] with the addition of exhaustivity.

3.4.1 Interpretation of Types. An es $A$ has binary conflict if there is an irreflexive symmetric binary relation $\#_{A}$ such that for all finite $X \subseteq A, X \in \mathrm{Con}_{A}$ iff for all $a_{1}, a_{2} \in X$, we have $\neg\left(a_{1} \#_{A} a_{2}\right)$. An $\operatorname{esp} A$ is positive (resp. negative) iff all its minimal events have positive (resp. negative) polarity. It is alternating iff for all $a_{1} \rightarrow A a_{2}$, we have $\operatorname{pol}_{A}\left(a_{1}\right) \neq \operatorname{pol}_{A}\left(a_{2}\right)$. It is sequential if $A$ has binary conflict, $\leq_{A}$ is tree-shaped (i.e. if $a_{1}, a_{2} \leq_{A} a$ then either $a_{1} \leq_{A} a_{2}$ or $a_{2} \leq_{A} a_{1}$ ) and conflict is local, i.e. if $x, x \cup\left\{a_{1}\right\}, x \cup\left\{a_{2}\right\} \in \mathscr{C}(A)$ and $x \cup\left\{a_{1}, a_{2}\right\} \notin \mathscr{C}(A)$ then $a_{1}$ and $a_{2}$ share the same antecedent.

The interpretation of types yields games that have a particular shape:

Definition 3.17. An arena is a $\sim$-game $\left(A, \kappa_{A}, \mathcal{H}_{A}, \cong_{A}, \cong_{A}^{+}, \cong_{A}^{-}\right)$with $A$ alternating and sequential. A +-arena is a non-empty positive arena $A$ s.t. all minimal events conflict pairwise, $\kappa_{A}(\emptyset)=-1$, and for all minimal $a \in A, \kappa_{A}(\{a\}) \geq 0$. A --arena is a negative arena such that $\kappa_{A}(\emptyset) \geq 0$.

Types will be interpreted as +-arenas. Note that if $A$ is a +-arena then $A^{\perp}$ is a --arena. If $N$ is a --arena and $H$ a Hilbert space, the down-shift $\downarrow_{H} N$ is the +-arena defined as $N$ prefixed with one new minimal positive event $\bullet$, with $Q(\bullet)=H$, and for $x \in \mathscr{C}(N), \kappa_{\downarrow_{H} N}(x \cup\{\bullet\})=\kappa_{N}(x)$. If $A, B$ are +-arenas, their sum $A \oplus B$ is defined as for $A \otimes B$, with added conflicts between all events of $A$ and $B$ and payoff function inherited - it generalizes to any arity in the obvious way.

Any +-arena decomposes (up to iso) as $A=\bigoplus_{i \in I_{A}} \downarrow_{H_{A, i}} N_{A, i}$, for $N_{A, i}$ some --arenas. Leveraging this we define two further constructions on +-arenas, the tensor and linear arrow:

$$
A \otimes B=\bigoplus_{(i, j) \in I_{A} \times I_{B}} \downarrow_{H_{A, i} \otimes H_{B, j}}\left(N_{A, i} \otimes N_{B, j}\right) \quad A \multimap B=\downarrow_{I}\left(\bigoplus_{i \in I_{A}} \downarrow_{H_{A, i}}\left(N_{A, i} \otimes B^{\perp}\right)\right)^{\perp} .
$$

We write $\lambda$ for the added minimal event of $A \multimap B$ as it stands for the evaluation to a $\lambda$-abstraction. For $x_{A}=\left\{\bullet_{i}\right\} \cup x_{A}^{-} \in \mathcal{E}_{A}$ and $x_{B}=\left\{\bullet_{j}\right\} \cup x_{B}^{-} \in \mathcal{E}_{B}$, we set $x_{A} \otimes x_{B}=\left\{\bullet_{(i, j)}\right\} \cup\left(x_{A}^{-} \| x_{B}^{-}\right) \in \mathcal{E}_{A \otimes B}$ - exhaustive configurations of $A \otimes B$ arise uniquely in this way. We also write $x_{A} \multimap x_{B}=$ $\left\{\lambda, \bullet_{i}\right\} \cup\left(x_{A}^{-} \| x_{B}\right) \in \mathcal{E}_{A \multimap B}$, and exhaustive configurations of $A \multimap B$ arise uniquely in this way.

The main type constructor left to interpret is $!(A \multimap B)$. We first introduce ! on --arenas.

Definition 3.18. The bang $! N$ of a --arena $N$ has underlying esp the infinitary $\|_{\mathbb{N}} N$, with inherited quantum annotations. Its symmetries rely on exchanging copy indices, we direct to [Clairambault et al. 2019] (Definition 6.3) for the definition and focus here on exhaustivity.

We set $\kappa ! N(\emptyset)=0$ as weakening is allowed on banged resources. If $x \in \mathscr{C}\left(\|_{\mathbb{N}} N\right)$ is non-empty, it is $\|_{i \in I} x_{i} \in \mathscr{C}\left(\|_{\mathbb{N}} N\right)$ with each $x_{i}$ non-empty. We then set $\kappa_{! N}\left(\|_{i \in I} x_{i}\right)=\kappa_{N}\left(x_{1}\right) \otimes \ldots \otimes \kappa_{N}\left(x_{|I|}\right)$ noting that the operation $\otimes$ on $\{-1,0,+1\}$ introduced in Section 3.1.2 is associative.

We do not have to define ! on arbitrary +-arenas since the matching type constructor only applies to linear functions. As the +-arenas corresponding to those has the form $\downarrow_{I} N$ for some --arena $N$, we set $!\left(\downarrow_{I} N\right)=\downarrow_{I}(! N)$. With this in place, we can give the interpretation of types:

$$
\begin{aligned}
\llbracket 1 \rrbracket & =\downarrow_{I} \varnothing & \llbracket A \multimap B \rrbracket & =\llbracket A \rrbracket \multimap \llbracket B \rrbracket & \llbracket A \otimes B \rrbracket & =\llbracket A \rrbracket \otimes \llbracket B \rrbracket \\
\llbracket \text { qbit } \rrbracket & =\downarrow_{\mathbb{C}^{2}} \varnothing & \llbracket !(A \multimap B) \rrbracket & =! \llbracket A \multimap B \rrbracket & \llbracket A^{\ell} \rrbracket & =\oplus_{n \in \mathbb{N}} \llbracket A^{\otimes n} \rrbracket
\end{aligned}
$$

yielding a +-arena $\llbracket A \rrbracket$ for any type $A$ of the quantum $\lambda$-calculus. We also write 1 for $\llbracket 1 \rrbracket=\downarrow_{I} \varnothing$. The rest of the paper does not involve --arenas, so from now on, arena will always refer to +-arena.

3.4.2 Interpretation of Terms. We will not refer to the details of the interpretation in the remainder of the exposition, so we only sketch it and refer the reader to [Clairambault et al. 2019].

The interpretation will take place in the subcategory of $\sim-Q C G[\mathcal{P}]$ having as objects, the arenas arising from the interpretation of types, and as morphisms from $A$ to $B$ the $\sim$-strategies 
$\sigma: S \rightarrow A^{\perp} \not>B$ that are negative (i.e. $S$ is negative); up to weak isomorphism. Let us write QA $[\mathcal{P}]$ for this subcategory. The monoidal product $\otimes$ of $\sim-Q C G[\mathcal{P}]$ does not transport to $\mathrm{QA}[\mathcal{P}]$ as it does not preserve arenas. However, the $\otimes$ operation above yields a premonoidal structure [Power and Robinson 1997] on $\mathrm{QA}[\mathcal{P}]$. The category $\mathrm{QA}[\mathcal{P}]$ also has coproducts given by $\oplus$.

Values are interpreted in a specific subcategory $\mathrm{QA}[\mathcal{P}]^{t}$ of $\mathrm{QA}[\mathcal{P}]$ with morphisms restricted to those $\sigma: S \rightarrow A^{\perp} \not 8 B$ which are thunkable: (1) for every minimal $s_{1}^{-} \in S$, there is exactly one $s_{2}^{+} \in S$ such that $s_{1} \rightarrow S s_{2}$, and $s_{2}$ maps to $B ;(2)$ in that case, $d_{Q_{\sigma}}\left[\left\{s_{1}\right\} ;\left\{s_{1}, s_{2}\right\}\right]=0$. In QA $[\mathcal{P}]^{t}, \otimes$ acts as a bifunctor; it is a symmetric monoidal category. The bang operation ! extends to a linear exponential comonad [Hyland and Schalk 2003] on the full sub-smc of QA $[\mathcal{P}]^{t}$ whose objects have only one minimal event with trivial Hilbert space. To interpret functions, we use the adjunction

$$
\mathrm{QA}[\mathcal{P}]^{t} \underset{A-\circ-}{\stackrel{I(-\otimes A)}{\underset{\perp}{\stackrel{\perp}{\rightleftarrows}}}} \mathrm{QA}[\mathcal{P}]
$$

familiar from closed Freyd categories [Power and Thielecke 1999]. For recursion we introduce a partial order on strategies with, for $\sigma: S \rightarrow A^{\perp} \not 8 B$ and $\sigma^{\prime}: S^{\prime} \rightarrow A^{\perp} \not 8 B$, setting $\sigma \sqsubseteq \sigma^{\prime}$ iff $S \subseteq S^{\prime}$ with the inclusion closed under symmetry and all components of $\sigma$ and $\sigma^{\prime}$ coinciding on $S$. This is a dcpo - with respect to [Clairambault et al. 2019], here we additionally observe that the lub of a directed set of strategies is exhaustive as exhaustivity deals with finite configurations. For quantum

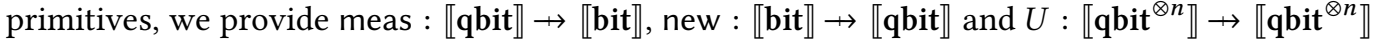
Dynamically, these strategies are straightforward: when exposed to a Opponent move on the left, they immediately play any Player move on the right. The quantum valuation of the corresponding configuration matches the standard CPM maps matching these operations (see Section 2.2.3).

The interpretation directly relies on the structure above. For the fragment of the language without formal parameters, the reader may find in [Clairambault et al. 2019] the full details along with computational adequacy. To this we must add the interpretation of the introduction rule for $\Gamma \vdash \mathcal{P} \mathrm{X} \cdot M: A$. Consider some finite set of parameters $\mathcal{P}$, such that $\mathrm{X} \in \mathcal{P}$. If $\sigma: S \rightarrow A^{\perp} \not \supset B$ is a strategy in $\mathrm{QA}[\mathcal{P}]$, we set $\mathrm{X} \cdot \sigma$ to share all components with $\sigma$, except for (with $x \in \mathscr{C}(X)$ )

$$
Q_{\mathrm{X} \cdot \sigma}(x)=\mathrm{X} \cdot \mathbf{Q}_{\sigma}(x)
$$

formally multiplying the polynomial $Q_{\sigma}(x)$ with X.

This concludes the interpretation of the parametrized quantum $\lambda$-calculus. We do not investigate adequacy; however it will be crucial that the interpretation commutes with substitution. For any $\rho \in[0,1]^{\mathcal{P}}$, the strict compact closed functor $-[\rho]: \operatorname{CPM}[\mathcal{P}] \rightarrow \mathrm{CPM}$ extends to a functor $-[\rho]: \mathrm{QA}[\mathcal{P}] \rightarrow \mathrm{QA}$ in the obvious way, preserving all operations on strategies. It follows that:

Proposition 3.19. Let $\Gamma \vdash \mathcal{P} M: A$, and $\rho \in[0,1]^{\mathcal{P}}$. Then, $\llbracket M \rrbracket[\rho]=\llbracket M[\rho] \rrbracket$.

\section{OBSERVATIONAL QUOTIENT}

The interpretation of the quantum $\lambda$-calculus in QA is not directly fully abstract, for a variety of reasons. Firstly, as usual and as emphasized earlier, game semantics display intermediate steps of computation which are not directly observable - to address that, we need to only compare strategies on their exhaustive configurations. Secondly, and more importantly, a strategy may realize one exhaustive configuration in potentially infinitely many ways: one must sum all these realizations.

\subsection{The Observational Sum}

For now, let us omit formal parameters and work with QA. We will reinstate them in Section 4.3.2.

Let $\sigma: S \rightarrow A^{\perp} \not \gamma B$ be a morphism in QA. Intuitively, to capture the observable behaviour of $\sigma$, for any exhaustive $x_{A} \| x_{B} \in \mathcal{E}_{A^{\perp \ngtr \beta} B}$ we would like to extract from $\sigma$ its weight. Setting 
wit $_{\sigma}\left(x_{A}, x_{B}\right)$ as the set of $x_{S} \in \mathscr{C}(S)+$-covered such that $\sigma x_{S}=x_{A} \| x_{B}$, we would like to sum

$$
\sigma_{x_{A}, x_{B}}=\sum_{x_{S} \in \text { wit }_{\sigma}\left(x_{A}, x_{B}\right)} Q_{\sigma}^{\vec{\sigma}}\left(x_{S}\right) \in \operatorname{CPM}\left(\mathcal{H}\left(x_{A}\right), \mathcal{H}\left(x_{B}\right)\right) .
$$

For the !-free fragment of the quantum $\lambda$-calculus, this would do just fine. In the presence of recursion and symmetry, two phenomena arise that need to be handled carefully.

4.1.1 Witnesses up to Symmetry. First, the set wit $_{\sigma}\left(x_{A}, x_{B}\right)$ above is too restrictive. In the presence of !, one must consider the behaviour of strategies up to symmetry. Accordingly, the weight $\sigma_{x_{A}, x_{B}}$ should account for witnesses matching $x_{A} \| x_{B}$ only up to symmetry. We set:

Definition 4.1. For $\sigma: S \rightarrow A^{\perp \ngtr \gamma} B$ and $x_{A} \| x_{B} \in \mathcal{E}_{A^{\perp} \chi_{B}}$, the witnesses for $x_{A} \| x_{B}$ up to symmetry comprises the configurations $x_{S} \in \mathscr{C}(S)$ +-covered, and such that $\sigma x_{S} \cong_{A^{\perp} \gamma_{B}}^{+} x_{A} \| x_{B}$.

We denote this set with wit ${ }_{\sigma}\left(x_{A}, x_{B}\right)$.

The reader may wonder why we consider the set of witnesses such that $\sigma x_{S}=x_{A}^{S} \| x_{B}^{S}$ is positively symmetry to $x_{A} \| x_{B}$, rather than merely symmetric. This "fixes" the Opponent copy indices: weakening positively symmetric to symmetric would bring us to count countably many times the same configuration as Opponent changes arbitrarily their copy indices.

Given $x_{A} \| x_{B} \in \mathcal{E}_{A^{\perp x_{B}}}$, our intention is to obtain $\sigma_{x_{A}, x_{B}}$ by summing $Q^{\rightarrow}\left(x_{S}\right)$ for each $x_{S} \in \operatorname{wit}_{\sigma}\left(x_{A}, x_{B}\right)$. But there is an issue: configurations $x_{S} \in$ wit $_{\sigma}\left(x_{A}, x_{B}\right)$ map to $x_{A}^{S} \| x_{B}^{S}$ only positively symmetric to $x_{A} \| x_{B}$, not equal. So $Q^{\rightarrow}\left(x_{S}\right) \in \operatorname{CPM}\left(\mathcal{H}\left(x_{A}^{S}\right), \mathcal{H}\left(x_{B}^{S}\right)\right)$, which is in general distinct from $\operatorname{CPM}\left(\mathcal{H}\left(x_{A}\right), \mathcal{H}\left(x_{B}\right)\right)$. For the sum to typecheck we must provide a way to canonically transport quantum weights between these isomorphic spaces.

Definition 4.2. Let $A$ be a quantum arena, and $x, x^{\prime} \in \mathscr{C}(A)$ be such that $x \cong x^{\prime}$. We define

$$
\gamma_{x, x^{\prime}}^{A}=\frac{1}{\left|x \cong_{A} x^{\prime}\right|} \sum_{\theta: x \cong_{A} x^{\prime}} \overline{\mathcal{H}_{A}(\theta)}: \operatorname{CPM}\left(\mathcal{H}_{A}(x), \mathcal{H}_{A}\left(x^{\prime}\right)\right)
$$

the symmetric transport from $x$ to $x^{\prime}$.

This is reminiscent of the construction of the symmetric tensor product in [Laird et al. 2013] as the equalizer of the permutation group for $n$-fold tensor products, obtained as their sum. A similar construction is also used for the exponential in [Pagani et al. 2014]. Ignoring for now convergence issues, the weight of $\sigma$ on configuration $x_{A} \| x_{B}$ is to be defined as

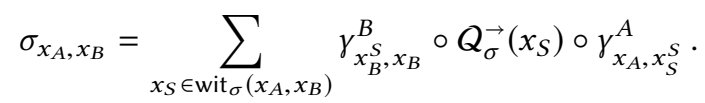

In fact, the more relevant notion is the weight of $\sigma$ on symmetry classes of exhaustive configurations. But the quantity given by the sum above is, as it turns out, not invariant under symmetry on $x_{A}, x_{B}$ : on non-canonical $x_{A}, x_{B}$, some witnesses are still accounted for several times.From now on, if $A$ is

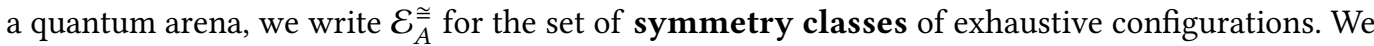
use $x, y$, etc to range over these symmetry classes. By definition of arenas, any such equivalence class comprises at least one canonical representative - from now on, for all $\mathrm{x} \in \mathcal{E}_{\bar{A}}^{\cong}$ we consider chosen one canonical representative, written $\underline{\mathrm{x}} \in \mathcal{E}_{A}$.

For $\sigma: S \rightarrow A^{\perp} \not 8 B$ and $\mathrm{x}_{A} \in \mathcal{E}_{A}^{\cong}, \mathbf{x}_{B} \in \mathcal{E}_{B}^{\cong}$ with wit ${ }_{\sigma}\left(\underline{\mathbf{x}}_{A}, \underline{\mathbf{x}}_{B}\right)$ finite, we set $\sigma_{\mathrm{x}_{A}, \mathrm{x}_{B}}$ as $\sigma_{\underline{\mathbf{x}}_{A}, \mathbf{x}_{B}}$.

4.1.2 D-Completion. If the set of witnesses is not finite, it is not clear that this sum converges. In fact, we shall see later that it does always converge, modulo a condition on strategies (visibility) to be introduced later. However, it will be convenient to give a formal status to these sums before they are known to converge. This may be done via D-completion [Zhao and Fan 2010]. 
We introduce briefly D-completion, following the presentation of [Pagani et al. 2014]. Given a partially ordered set $(P, \leq)$, a subset $S$ is Scott-closed if it is down-closed, and for every directed $I \subseteq S$, if the lub $\vee I$ exists in $P$, then $\vee I \in S$. A monotone function $f: P \rightarrow Q$ between partially ordered sets is Scott-continuous if it preserves all existing least upper bounds of directed subsets The set of all Scott-closed subsets of $P$ forms a directed complete partial order (dcpo), and the D-completion $\bar{P}$ of $P$ is then defined as its smallest sub-dcpo comprising the down-closure $[p]$ for each $p \in P$. Then $\bar{P}$ is a dcpo, and there is a canonical Scott-continuous injection $\iota: P \rightarrow \bar{P}$ through which we regard $P$ as a subset of $\bar{P}$. If $P$ is a bounded directed complete partial order, then $P$ is an initial subset of $\bar{P}$, i.e. the only new elements added by completion are "at infinity".

Following [Pagani et al. 2014] we now complete CPM into a dcpo-enriched category $\overline{\mathbf{C P M}}$. For any two Hilbert spaces $H$ and $K, \operatorname{CPM}(H, K)$ is partially ordered via the Löwner order. We set $\overline{\mathbf{C P M}}(H, K)=\overline{\mathbf{C P M}(H, K)}$ the corresponding D-completion. All operations in CPM are Scottcontinuous with respect to the Löwner order, and as such extend to $\overline{\mathrm{CPM}}$ canonically. If $\left(f_{i}\right)_{i \in I}$ is any family of completely positive maps $f_{i} \in \mathbf{C P M}(H, K)$, then the infinite sum $\sum_{i \in I} f_{i} \in \overline{\mathbf{C P M}}(H, K)$ is always defined, as the lub of the directed set comprising $\sum_{i \in F} f_{i} \in \mathbf{C P M}(H, K)$ for any $F$ a finite subset of $I$. Composition and scalar multiplication being linear and Scott-continuous, they distribute over sums. Finally, for $\sigma: S \rightarrow A^{\perp} \not 8 B$ in $\mathrm{QA}$ and $\mathrm{x}_{A} \in \mathcal{E}_{\bar{A}}^{\cong}, \mathrm{x}_{B} \in \mathcal{E}_{B}^{\cong}$, we temporarily define

$$
\sigma_{\mathrm{x}_{A}, \mathrm{x}_{B}}=\sum_{x_{S} \in \mathrm{wit}_{\sigma}\left(\underline{\mathrm{x}}_{A}, \underline{\mathrm{x}}_{B}\right)} \gamma_{x_{B}^{S}, \underline{\mathrm{x}}_{B}}^{B} \circ Q_{\sigma}^{\rightarrow}\left(x_{S}\right) \circ \gamma_{\underline{\mathbf{x}}_{A}, x_{A}^{S}}^{A} \in \overline{\mathbf{C P M}}\left(\mathcal{H}\left(\underline{\mathbf{x}}_{A}\right), \mathcal{H}\left(\underline{\mathbf{x}}_{B}\right)\right),
$$

although we will see shortly that (for visible strategies) only finite elements of $\overline{\mathbf{C P M}}$ are reached. Note that this definition only covers strategies in QA (without parameters). We postpone defining the observational sum of strategies in $\mathrm{QA}[\mathcal{P}]$ until we have convergence without parameters.

\subsection{Congruence of the Observational Sum}

The observational sum introduced above induces an equivalence relation on strategies: for $\sigma$ : $S \rightarrow A^{\perp} \not \partial B$ and $\sigma^{\prime}: S^{\prime} \rightarrow A^{\perp \not \gamma} B$, we set $\sigma \equiv \sigma^{\prime}$ iff for all $\mathrm{x}_{A} \in \mathcal{E}_{A}^{\cong}$ and $\mathrm{x}_{B} \in \mathcal{E}_{B}^{\cong}$, we have $\sigma_{\mathrm{x}_{A}, \mathrm{x}_{B}}=\sigma_{\mathrm{x}_{A}, \mathrm{x}_{B}}^{\prime}$. We shall prove that $\mathrm{QA}$, considered up to $\equiv$, is fully abstract for the quantum $\lambda$-calculus. But for that, we must first prove that quotienting QA by $\equiv$ yields a model, i.e. that $\equiv$ is preserved by all operations on strategies. The critical point is to prove that composition preserves $\equiv$, which boils down to: for any $\sigma: S \rightarrow A^{\perp} \not 8 B, \tau: T \rightarrow B^{\perp} \not 8 C, \mathrm{x}_{A} \in \mathcal{E}_{\bar{A}}^{\simeq}$ and $\mathrm{x}_{C} \in \mathcal{E}_{B}^{\underline{\underline{\rho}}}$, we have

$$
(\tau \odot \sigma)_{\mathbf{x}_{A}, \mathrm{x}_{C}}=\sum_{\mathrm{x}_{B} \in \mathcal{E}_{\bar{B}}^{\underline{\tilde{x}}}} \tau_{\mathrm{x}_{B}, \mathrm{x}_{C}} \circ \sigma_{\mathrm{x}_{A}, \mathrm{x}_{B}} \in \overline{\mathbf{C P M}}\left(\mathcal{H}\left(\underline{\mathbf{x}}_{A}\right), \mathcal{H}\left(\underline{\mathbf{x}}_{C}\right)\right)
$$

This is a very challenging property to prove, involving subtle manipulations of games with symmetry in combination with manipulations of the quantum valuations. Intuitively (but slightly misleadingly, see Section 4.2.2).we must establish a bijection between witnesses $x_{T} \odot x_{S} \in$ wit $_{\sigma}\left(\underline{\mathbf{x}}_{A}, \underline{\mathbf{x}}_{C}\right)$ on the one hand, and triples $\left(\mathrm{x}_{B}, x_{S}, x_{T}\right)$ with $\mathrm{x}_{B} \in \mathcal{E}_{B}^{\cong}, x_{S} \in$ wit $_{\sigma}\left(\underline{\mathrm{x}}_{A}, \underline{\mathrm{x}}_{B}\right), x_{T} \in$ wit $_{\tau}\left(\underline{\mathrm{x}}_{B}, \underline{\mathrm{x}}_{C}\right)$ on the other hand, in such a way that the quantum valuations are preserved.

4.2.1 Deadlock-free Composition. The very notation introduced in Proposition 3.6 carries, for configurations $x_{T} \odot x_{S} \in \mathscr{C}(T \odot S)$, the data of $x_{S} \in \mathscr{C}(S)$ and $x_{T} \in \mathscr{C}(T)$ - it might seem that the desired bijection should simply follow this. Recall that configurations $x_{T} \odot x_{S} \in \mathscr{C}(T \odot S)$ are in one-to-one correspondence with pairs of configurations $x_{S} \in \mathscr{C}(S)$ (write $\sigma x_{S}=x_{A}^{S} \| x_{B}^{S}$ ) and $x_{T} \in \mathscr{C}(T)$ (write $\tau x_{T}=x_{B}^{T} \| x_{C}^{T}$ ) (1) matching on $B\left(\right.$ i.e. $x_{B}^{S}=x_{B}^{T}$ ); and (2) causally secured, in the sense that their synchronization introduces no deadlock. The item (2) is an obstacle to our bijection, corresponding to a fundamental difference between games models and relational-like models. 
Earlier work [Castellan 2017; Castellan et al. 2018, 2015] has established that the concept of visibility, inspired from traditional game semantics [Abramsky and McCusker 1996], induces a deadlock-free composition. It only involves the bare causal structure of strategies. If $\sigma: S \rightarrow A^{\perp} \not 8 B$ is a strategy, a grounded causal chain (gcc) in $S$ is an immediate causal chain $\rho=s_{0} \rightarrow S s_{1} \rightarrow S$ $\ldots \rightarrow S s_{n}$ in $S$, such that $s_{0}$ is minimal in $S$. We identify $\rho$ with the set $\left\{s_{0}, \ldots, s_{n}\right\}$, totally ordered by $\leq_{S}$. We write $\operatorname{gcc}(S)$ for the set of $g c c s$ in $S$. We define visible strategies:

Definition 4.3. A strategy $\sigma: S \rightarrow A^{\perp} \not \supset B$ is visible iff for any $\rho \in \operatorname{gcc}(S), \sigma \rho \in \mathscr{C}\left(A^{\perp} \| B\right)$.

Gccs mapping correctly to the game are strongly related with the traditional game semantical notion of $P$-views [Castellan et al. 2015], capturing branches of sequential purely functional programs. One can read visibility as stating that all gccs are "valid P-views", so that overall the strategy may be regarded as patching together all these P-views, expressing how they branch causally, non-deterministically, and merge causally. Visible strategies include both sequential and parallel interpretations of pure functional programs [Castellan et al. 2015]. Furthermore, we have:

Lemma 4.4. let $\sigma: S \rightarrow A^{\perp} \not \gamma B, \tau: T \rightarrow B^{\perp} \not \supset C$ be visible strategies. Take $x_{S} \in \mathscr{C}(S)$ with $\sigma x_{S}=x_{A}^{S} \| x_{B}^{S}$ and $x_{T} \in \mathscr{C}(T)$ with $\tau x_{T}=x_{B}^{T} \| x_{C}^{T}$, and $\theta: x_{B}^{S} \cong_{B} x_{B}^{T}$. Then, the induced

$$
\varphi: x_{S}\left\|x_{C}^{T} \stackrel{\sigma \| x_{C}^{T}}{\simeq} x_{A}^{S}\right\| x_{B}^{S}\left\|x_{C}^{T} \stackrel{x_{A}^{S}\|\theta\| x_{X}^{T}}{\cong} x_{A}^{S}\right\| x_{B}^{T}\left\|x_{C}^{T} \stackrel{x_{A}^{S} \| \tau}{\simeq} x_{A}^{S}\right\| x_{T}
$$

is secured - in other words, for $\theta=\mathrm{id}_{x_{B}}$, condition (2) of Proposition 3.6 is redundant.

Conceptually, this is strongly connected with Melliès' observation that innocent strategies in asynchronous games are positional [Melliès 2006]. Technically, this generalizes the deadlock-free lemma of [Castellan et al. 2018], covering the case of synchronization up to symmetry. Visible strategies are stable under all relevant constructions. From now on we consider that all strategies are visible - the categories QA, QA $[\mathcal{P}]$ now assume visibility as well.

4.2.2 Synchronization up to Symmetry. With deadlocks put aside, we now examine the main issue in proving congruence. Given (visible) strategies $\sigma: S \rightarrow A^{\perp} \not \gamma B, \tau: T \rightarrow B^{\perp} \not 8 C$, and $\mathrm{x}_{A} \in \mathcal{E}_{\bar{A}}$, $\mathrm{x}_{C} \in \mathcal{E}_{\bar{C}}^{\cong}$, we first fix $\mathrm{x}_{B} \in \mathcal{E}_{B}^{\cong}$ and examine the sum

$$
\tau_{\mathrm{x}_{B}, \mathrm{x}_{C}} \circ \sigma_{\mathrm{x}_{A}, \mathrm{x}_{B}}=\sum_{x_{S} \in \mathrm{wit}_{\sigma}\left(\underline{\mathrm{x}}_{A}, \underline{\mathrm{x}}_{B}\right)} \sum_{\left.x_{T} \in \mathrm{wit}_{\tau} \underline{\underline{x}}_{B}, \underline{\mathbf{x}}_{C}\right)} \gamma_{x_{C}^{T}, \underline{x}_{C}}^{C} \circ Q_{\tau}^{\rightarrow}\left(x_{T}\right) \circ \gamma_{x_{B}^{S}, x_{B}^{T}}^{B} \circ Q_{\sigma}^{\rightarrow}\left(x_{S}\right) \circ \gamma_{\underline{x}_{A}, x_{A}^{S}}^{A} .
$$

using that $\gamma_{\underline{x}_{B}, x_{B}^{T}}^{B} \circ \gamma_{x_{B}^{S}, \underline{x}_{B}}^{B}=\gamma_{x_{B}^{S}, x_{B}^{T}}^{B}$. Unfolding the definition of $\gamma$ in the middle, we are brought to consider a sum ranging over $x_{S} \in$ wit $_{\sigma}\left(\underline{\mathrm{x}}_{A}, \underline{\mathrm{x}}_{B}\right), x_{T} \in \mathrm{wit}_{\tau}\left(\underline{\mathrm{x}}_{B}, \underline{\mathrm{x}}_{C}\right)$, with a mediating symmetry $\theta_{B}: x_{B}^{S} \cong_{B} x_{B}^{T}$. In fact, a crucial aspect of games with symmetry [Castellan et al. 2019] is that in this case, it is always possible to find symmetric $y_{S} \in \mathscr{C}(S)$ and $y_{T} \in \mathscr{C}(T)$ matching on the nose.

Lemma 4.5. Let $\sigma: S \rightarrow A^{\perp} \not>B, \tau: T \rightarrow B^{\perp} \not>C$ be strategies. Consider furthermore $x_{S} \in \mathscr{C}(S)$ with $\sigma x_{S}=x_{A}^{S} \| x_{B}^{S}, x_{T} \in \mathscr{C}(T)$ with $\tau x_{T}=x_{B}^{T} \| x_{C}^{T}$, and $\theta: x_{B}^{S} \cong_{B} x_{B}^{T}$.

Then, there are $\varphi_{S}: x_{S} \cong_{S} y_{S}, \varphi_{T}: x_{T} \cong_{T} y_{T}$ such that $\sigma y_{S}=y_{A} \| y_{B}$ and $\tau y_{T}=y_{B} \| y_{C}$ match on $B$ on the nose, along with $\theta_{C}: y_{C} \cong_{C} x_{C}^{T}$ and $\theta_{A}: x_{A}^{S} \cong_{A} y_{A}$ such that

$$
Q_{\tau}\left(x_{T}\right) \circ \widehat{\mathcal{H}(\theta)} \circ Q_{\sigma}\left(x_{S}\right)=\overline{\mathcal{H}\left(\theta_{C}\right)} \circ Q_{\tau}^{\rightarrow}\left(y_{T}\right) \circ Q_{\sigma}\left(y_{S}\right) \circ \overline{\mathcal{H}\left(\theta_{A}\right)} .
$$

This puts together Lemma 3.23 of [Castellan et al. 2019], preservation of quantum valuations under symmetry, and Lemma 4.4. This goes in the right direction, giving a qualitative equivalence between pairs of configurations of $\sigma$ and $\tau$ matching up to symmetry and pairs matching on the nose. However, to prove congruence one must refine it to a quantitative correspondence. This is 


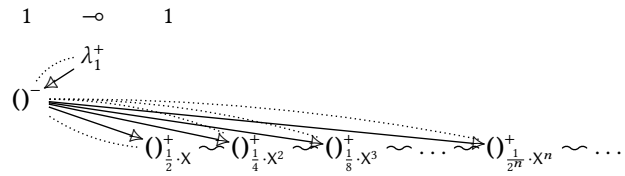

Fig. 8. The interpretation of letrec $f^{1 \multimap 1} x^{1}=X \cdot\left(\frac{1}{2} \cdot\right.$ skip $\left.+\frac{1}{2} \cdot(f x)\right)$ in $: 1 \multimap 1$

quite subtle, using in an essential way the hypothesis that each symmetry class of configurations has a canonical representative. Details are omitted by lack of space.

With this we can prove Equation 1, from which it follows that $\equiv$ is stable under composition. It is easy to prove that the other constructions on strategies preserve $\equiv$ as well. Hence:

Proposition 4.6. There is a category $\mathrm{QA} / \equiv$ whose objects are interpretation of types and morphisms from $A$ to $B$ are strategies $\sigma: S \rightarrow A^{\perp} \not 2 B$, considered up to $\equiv$.

\subsection{Convergence of the Observational Sum}

With the help of Equation 1 we can prove that the observational sum always converges in CPM.

4.3.1 Convergence for QA. As it stands, the quantum games model already has good convergence properties. Indeed we mentioned in Section 3.2.1 that quantum annotations, reorganized as CPM maps from (spaces generated by) negative events to (spaces generated by) positive events, yield superoperators. This convergence is also embodied by the following simpler property:

Lemma 4.7. Let $\sigma: S \rightarrow 1^{\perp} \| 1$ in QA. Then, $\sigma_{\{()\} \|\{()\}} \in[0,1]$.

This is an immediate consequence of the monotone condition for quantum strategies, which in the absence of quantum spaces boils down to the conditions on probabilistic strategies [Winskel 2015]. Using this, we prove convergence by exploiting that we can "trace out" any strategy:

Proposition 4.8. For any $\sigma: A \rightarrow B$ in $\mathrm{QA}$ and $\mathrm{x}_{A} \in \mathcal{E}_{\bar{A}}^{\cong}, \mathrm{x}_{B} \in \mathcal{E}_{B}^{\cong}, \sigma_{\mathrm{x}_{A}, \mathrm{x}_{B}} \in \operatorname{CPM}\left(\mathcal{H}\left(\underline{\mathbf{x}}_{A}\right), \mathcal{H}\left(\underline{\mathrm{x}}_{B}\right)\right)$.

Proof. To prove this, we show that there is a constant $N_{\mathrm{x}_{A}, \mathrm{x}_{B}} \in \mathbb{N}$ and quantum strategies

$$
\beta_{A} \in \mathrm{QA}(1, A) \quad \beta_{B} \in \mathrm{QA}(B, 1)
$$

such that for any $f \in \overline{\mathbf{C P M}}\left(\mathcal{H}\left(\underline{\mathbf{x}}_{A}\right), \mathcal{H}\left(\underline{\mathbf{x}}_{B}\right)\right)$, writing $\operatorname{tr}(f)$ for $\bigvee_{\emptyset, \emptyset} f \in \overline{\mathbf{C P M}}(I, I)$,

$$
\operatorname{tr}(f) \leq N_{\mathrm{x}_{A}, \mathrm{x}_{B}}\left(\beta_{B}\right)_{\mathrm{x}_{B},\{()\}} \circ f \circ\left(\beta_{A}\right)_{\{()\}, \mathrm{x}_{A}} .
$$

Because all objects in QA are generated by types, we may define $\beta_{A}$ and $\beta_{B}$ with the syntax of the quantum $\lambda$-calculus - those are $\beta_{A}=\Uparrow_{\underline{x}_{A}}^{A}$ and $\beta_{B}=\Downarrow_{\underline{x}_{B}}^{B}$ to be defined in Section 5.1, with all formal parameters set to 1 . Instantiating this with $\sigma_{\mathrm{x}_{A}, \mathrm{x}_{B}} \in \overline{\mathrm{CPM}}\left(\mathcal{H}\left(\underline{\mathrm{x}}_{A}\right), \mathcal{H}\left(\underline{\mathrm{x}}_{B}\right)\right)$, we obtain

$$
\operatorname{tr}\left(\sigma_{\mathrm{x}_{A}, \mathrm{x}_{B}}\right) \leq N_{\mathrm{x}_{A}, \mathrm{x}_{B}}\left(\beta_{B}\right)_{\mathrm{x}_{B},\{()\}} \circ \sigma_{\mathrm{x}_{A}, \mathrm{x}_{B}} \circ\left(\beta_{A}\right)_{\{()\}, \mathrm{x}_{A}}
$$

but by Equation 1, $\left(\beta_{B}\right)_{\mathrm{x}_{B},\{()\}} \circ \sigma_{\mathrm{x}_{A}, \mathrm{x}_{B}} \circ\left(\beta_{A}\right)_{\mathrm{x}_{A},\{()\}}$ is a term in the $\operatorname{sum}\left(\beta_{B} \odot \sigma \odot \beta_{A}\right)_{\{()\},\{()\} \text {, which }}$ is in $[0,1]$ by Lemma 4.7. So $\operatorname{tr}\left(\sigma_{\mathrm{x}_{A}, \mathrm{x}_{B}}\right) \leq N_{\mathrm{x}_{A}, \mathrm{x}_{B}}$, therefore $\sigma_{\mathrm{x}_{A}, \mathrm{x}_{B}}$ must be finite.

4.3.2 Convergence for $\mathrm{QA}[\mathcal{P}]$. We now aim to prove a similar convergence property for strategies in QA $[\mathcal{P}]$, in the presence of formal parameters. However, an issue immediately arises: for $\sigma: S \rightarrow$ $A^{\perp \not \gamma} B$ in $\mathrm{QA}[\mathcal{P}]$ with $\mathrm{x}_{A} \in \mathcal{E}_{\bar{A}}^{\simeq}, \mathrm{x}_{B} \in \mathcal{E}_{B}^{\cong}$, it is not the case that $\sigma_{\mathrm{x}_{A}, \mathrm{x}_{B}} \in \mathrm{CPM}[\mathcal{P}]\left(\mathcal{H}\left(\underline{\mathrm{x}}_{A}\right), \mathcal{H}\left(\underline{\mathrm{x}}_{B}\right)\right)$

Figure 8 illustrates the issue (a purely probabilistic example suffices) - the figure uses wiggly lines to indicate that all events occurring in the third row are in pairwise conflict with each other. 
There are infinitely many witnesses for the exhaustive configuration $x=\left\{\lambda^{+},()^{-},()^{+}\right\}$, yielding $\sigma_{\mathrm{x}}=\sum_{n=1}^{+\infty} \frac{1}{2^{n}} \mathrm{X}^{n}$ where $\sigma$ is the strategy of Figure 8 - while each witness contributes a polynomial, the infinite sum may involve infinitely many monomials. Therefore, in general, when summing all witnesses we must move from multivariate polynomials to multivariate power series.

For $H, K$ in $\mathrm{Hilb}$, a $\mathrm{CPM}(H, K)$-valued power series with parameters in $\mathcal{P}=\left\{\mathrm{X}_{1}, \ldots, \mathrm{X}_{n}\right\}$ is

$$
\sum_{i \in I} f_{i} \mathrm{X}_{1}^{\alpha_{i, 1}} \ldots \mathrm{X}_{n}^{\alpha_{i, n}}
$$

a formal sum with $I$ countable, and for all $i \in I, f_{i} \in \mathrm{CPM}(H, K)$. Its domain of convergence is the set of $\rho \in[0,1]^{\mathcal{P}}$ such that the sum $\sum_{i \in I} f_{i} \rho\left(\mathrm{X}_{1}\right)^{\alpha_{i, 1}} \ldots \rho\left(\mathrm{X}_{n}\right)^{\alpha_{i, n}}$ converges in $\operatorname{CPM}(H, K)$ Observe that if the sum converges with the summands being added in some order, then it absolutely converges. This is because through the Choi-Jamiolkowski we are summing positive operators, on which the trace is a norm; and if the sum converges, so does the trace. In this way, all infinite sums considered in this paper are invariant under reordering of the summands.

We write $\operatorname{CPM}\{\mathcal{P}\}(H, K)$ for the set of $\mathbf{C P M}(H, K)$-valued power series with parameters in $\mathcal{P}=\left\{\mathrm{X}_{1}, \ldots, \mathrm{X}_{n}\right\}$ whose domain of convergence is $[0,1]^{n}$.

Proposition 4.9. If $\sigma: S \rightarrow A^{\perp \not \gamma} B$ is a strategy in $\mathrm{QA}[\mathcal{P}], \mathrm{x}_{A} \in \mathcal{E}_{\bar{A}}^{\cong}$ and $\mathrm{x}_{B} \in \mathcal{E}_{\bar{B}}^{\cong}$, then

$$
\sigma_{\mathrm{x}_{A}, \mathrm{x}_{B}} \in \operatorname{CPM}\{\mathcal{P}\}\left(\mathcal{H}\left(\underline{\mathrm{x}}_{A}\right), \mathcal{H}\left(\underline{\mathrm{x}}_{B}\right)\right) .
$$

Proof. For each monomial $\mathrm{m}_{i}=\mathrm{X}_{1}^{\alpha_{i, 1}} \ldots \mathrm{X}_{n}^{\alpha_{i, n}}$, the coefficient $f_{i}$ is the sum of all coefficients attached to $\mathrm{m}_{i}$ in $Q_{\sigma}\left(x_{S}\right)$ for some $x_{S} \in$ wit $_{\sigma}\left(\underline{\mathrm{x}}_{A}, \underline{\mathrm{x}}_{B}\right)$. Writing $\rho_{1}\left(\mathrm{X}_{i}\right)=1$ for all $\mathrm{X}_{i}, f_{i}$ is obtained as a limit of finite sums, all of which are less (for the Löwner order) than $\left(\sigma\left[\rho_{1}\right]\right)_{\mathrm{x}_{A}, \mathrm{x}_{B}}$. By Proposition 4.8, $\left(\sigma\left[\rho_{1}\right]\right)_{\mathrm{x}_{A}, \mathrm{x}_{B}}$ is in $\operatorname{CPM}\left(\mathcal{H}\left(\underline{\mathrm{x}}_{A}\right), \mathcal{H}\left(\underline{\mathrm{x}}_{B}\right)\right)$. Hence, $f_{i} \in \operatorname{CPM}\left(\mathcal{H}\left(\underline{\mathrm{x}}_{A}\right), \mathcal{H}\left(\underline{\mathrm{x}}_{B}\right)\right)$.

Now, for $\rho \in[0,1]^{\mathcal{P}}$ we have $\sigma_{\mathrm{x}_{A}, \mathrm{x}_{B}}[\rho]=(\sigma[\rho])_{\mathrm{x}_{A}, \mathrm{x}_{B}} \in \mathbf{C P M}\left(\mathcal{H}\left(\underline{\mathbf{x}}_{A}\right), \mathcal{H}\left(\underline{\mathbf{x}}_{B}\right)\right)$ by Prop. 4.8.

The category $\operatorname{CPM}\{\mathcal{P}\}$ has objects Hilbert spaces, morphisms power series in $\operatorname{CPM}\{\mathcal{P}\}(H, K)$. Composition is defined as that of $\mathrm{CPM}[\mathcal{P}]$. The proof of Equation 1 applies transparently, showing that for $\sigma: S \rightarrow A^{\perp} \not \mathcal{B} B$ and $\tau: T \rightarrow B^{\perp} \not \mathcal{Z} C$ in $\mathrm{QA}[\mathcal{P}], \mathrm{x}_{A} \in \mathcal{E}_{A}^{\cong}, \mathrm{x}_{C} \in \mathcal{E}_{C}^{\cong}$,

$$
(\tau \odot \sigma)_{\mathbf{x}_{A}, \mathrm{x}_{C}}=\sum_{\mathrm{x}_{B} \in \mathcal{E}_{\bar{B}}^{\underline{\simeq}}} \tau_{\mathrm{x}_{B}, \mathrm{x}_{C}} \circ \sigma_{\mathrm{x}_{A}, \mathrm{x}_{B}} \in \mathbf{C P M}\{\mathcal{P}\}\left(\mathcal{H}\left(\underline{\mathbf{x}}_{A}\right), \mathcal{H}\left(\underline{\mathbf{x}}_{C}\right)\right) .
$$

Now, we are equipped to attack the full abstraction proof.

\section{FULL ABSTRACTION FOR GAMES AND QUANTUM RELATIONS}

Let us motivate the constructions to come, aiming for full abstraction. Assume we have two terms $\vdash M, N: A$ for some type $A$, which have a different interpretation in $\mathrm{QA} / \equiv$. This means that there is some $\mathrm{x} \in \mathcal{E}_{\llbracket}^{\cong} \underline{\underline{\underline{a}}}$ such that $\llbracket M \rrbracket_{\mathrm{x}} \neq \llbracket N \rrbracket_{\mathrm{x}}$. We must use this information to separate $M$ and $N$, by producing a context $C[-]$ which will somehow extract from $M$ and $N$ their behaviour on $\mathrm{x}$.

\subsection{Testing Terms}

Performing the extraction is the purpose of the testing terms. We start by presenting the intuition behind their construction, in the probabilistic case. For any $p, q \in[0,1]$, consider $M_{p, q}$ defined as

$$
f: !(1 \multimap \text { bit }) \vdash \text { if } f \text { (skip) then (if } f \text { (skip) then }(p \cdot \text { skip) else }(q \cdot \text { skip })) \text { else } \perp: 1
$$

where divergence $\perp$ is definable through recursion. Figure 9 displays, on the left of each composition, the only two exhaustive configurations of $\llbracket M_{p, q} \rrbracket$. The valuation of the configurations appears as a subscript for both last moves. We omit the copy indices coming from the ! to avoid clutter. 


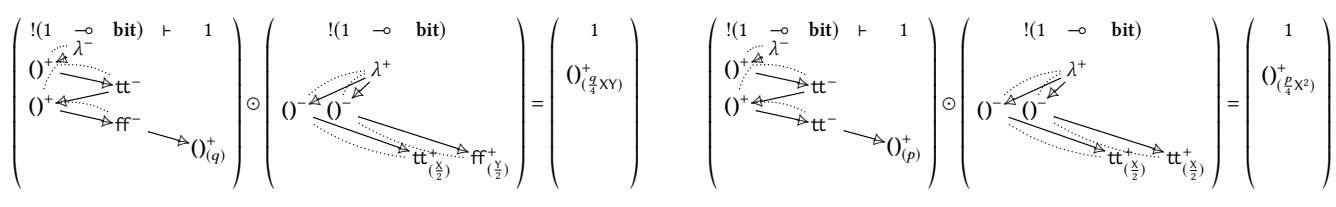

Fig. 9. Computing $\llbracket T \rrbracket \odot \llbracket M_{p, q} \rrbracket \equiv()_{\left(\frac{q}{4} X Y+\frac{p}{4} Y^{2}\right)}^{+}$

$$
\begin{aligned}
& \Downarrow_{\{(0)\}}^{1}(v)=v \\
& \Downarrow_{x_{A} \| \emptyset}^{A \oplus B}(v)=\operatorname{match} v \text { with }\left(y^{A}: \Downarrow_{x_{A}}^{A}(y) \mid w^{B}: \perp\right) \\
& \Downarrow_{\emptyset \| x_{B}}^{A \oplus B}(v)=\operatorname{match} v \text { with }\left(y^{A}: \perp \mid w^{B}: \Downarrow_{x_{B}}^{B}(w)\right) \\
& \Downarrow_{x_{A} \otimes x_{B}}^{A \otimes B}(v)=\text { let } y^{A} \otimes w^{B}=v \text { in }\left(\Downarrow_{x_{A}}^{A}(y) \otimes \Downarrow_{x_{B}}^{B}(w)\right) \\
& \Downarrow_{x_{A} \rightarrow x_{B}}^{A \rightarrow B}(f)=\Downarrow_{x_{B}}^{B}\left(f\left(\Uparrow_{x_{A}}^{A}\right)\right) \\
& \Downarrow_{\|_{i \in I}\left(x_{i}\right)}^{!(A-B)}(f)=\Downarrow_{x_{1}}^{A-\circ B}(f) ; \ldots ; \Downarrow_{x_{|I|}}^{A-B}(f) \\
& \Downarrow_{x_{n+1} \otimes \ldots \otimes x_{1}}^{A^{\ell}}(t:: u)=\Downarrow_{x_{n+1}}^{A}(t) ; \Downarrow_{x_{n} \otimes \ldots \otimes x_{1}}^{A^{\ell}}(u) \\
& \Downarrow_{\{0\}}^{A^{\ell}}([])=\text { skip } \quad \Downarrow_{\{0\}}^{A^{\ell}}(t:: u)=\perp
\end{aligned}
$$

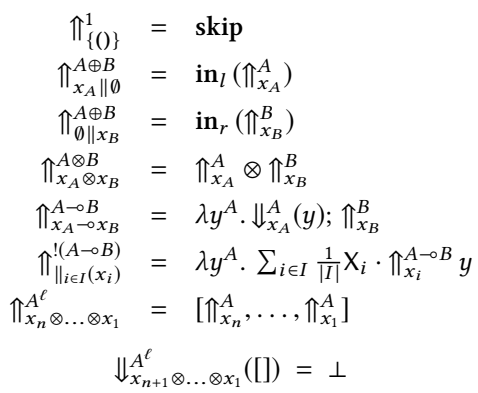

Fig. 10. Testing and generating terms for the classical fragment

How can one build a context that separates the two? Clearly, there is no purely deterministic context that separates $M_{0, \frac{1}{2}}$ and $M_{0, \frac{1}{3}}$ because they only differ on a configuration (shown at the left hand side of Figure 9) where the argument function behaves non-uniformly. To separate them, one can instead use a probabilistic function $T=\lambda x \cdot \frac{X}{2} \cdot t t+\frac{Y}{2} \cdot f f$ for well-chosen $X, Y \in[0,1]$.

5.1.1 Classical Testing Terms. In general, given $\mathrm{x} \in \mathcal{E}_{\bar{A}}^{\cong}$ on which two terms $M$ and $N$ differ, one can build a term that can replay $\mathrm{x}$ with $M$ and $N$, targetting the distinguishing behaviour. In particular, if $\mathrm{x}$ has multiple calls to a function, the corresponding test will feature an adequately weighted probabilistic sum over the behaviours performed by the context in the different copies of that call in $\mathrm{x}$, so that the test will be able to interact with tested terms as prescribed by $\mathrm{x}$.

Let us now show how these testing terms are defined for classical types, postponing for now the quantum case. For any classical type $A$ and $x \in \mathcal{E}_{\llbracket A \rrbracket}$, we define mutually inductively two terms

$$
v: A \vdash \Downarrow_{x}^{A}(v): 1 \quad \vdash \Uparrow_{x}^{A}: A,
$$

the testing term $\Downarrow_{x}^{A}(v)$ (with free variable $v: A$ ) and the generating term $\Uparrow_{x}^{A}$ (we leave implicit the annotation of $\vdash$ with the set of formal parameters in typing judgments). The definition is given in Figure 10. These testing terms for classical types are conceptually close to those of [Ehrhard et al. 2014]; they differ mainly in that our language is call-by-value whereas probabilistic PCF is call-by-name. Our notation is inspired from that used in normalization by evaluation [Dybjer and Filinski 2000], which uses analogous combinators. Generation on type $(A \multimap B)$ involves a probabilistic sum, each clause weighted by a fresh parameter to be instantiated later.

If $M, N: A$ differ on symmetry class $\mathrm{x}$ with representative $x, \Downarrow_{x}^{A}$ will replay $x$ with $M$ and $N$, yielding configurations of $\Downarrow_{x}^{A}(M)$ and $\Downarrow_{x}^{A}(N)$ of ground type with distinct weights. For instance, the terms $\lambda f . M_{0, \frac{1}{2}}$ and $\lambda f . M_{0, \frac{1}{3}}$ only differ through the valuation they assign to the configuration $x$ where the argument returns tt once, and $\mathrm{ff}$ once. The matching testing term is

$$
\Downarrow_{x}^{!(1-\mathrm{obit})-01}=\lambda g^{!(1-\mathrm{obit})-\circ 1} \cdot g \Uparrow_{\{()\}-\{\{\mathrm{tt}\} \|\{()\} \multimap\{\mathrm{ff}\}}^{!(1-\mathrm{bit})}=\lambda g^{!(1-\mathrm{obit})-\circ 1} \cdot g T
$$


where, up to simple conversion, $T=\frac{X}{2} \cdot t t+\frac{Y}{2} \cdot f f$ is the term above. Composition between $\lambda f . M$ and $\Downarrow_{x}^{!(1-\mathrm{bit})-\circ 1}$ amounts to composition between $M$ and $T$ - the left hand side of Figure 9 shows a configuration of that composition successfully replaying the configuration of interest.

However, there is a complication. Although the testing term $T$ is designed to replay one particular configuration, it might successfully interact with $M_{p, q}$ in other ways too. For instance, we show in the right hand side of Figure 9 another successful composition of $M_{p, q}$ and $T$, where the two calls to $T$ select the same branch and both return tt. This composition also contributes to the probability of convergence of $M_{p, q} T$, which is $\frac{q}{4} \mathrm{XY}+\frac{p}{4} \mathrm{Y}^{2}$ for all $p, q, \mathrm{X}, \mathrm{Y} \in[0,1]$. If $\mathrm{X}$ and $\mathrm{Y}$ are chosen poorly, $T$ might fail to distinguish terms. For instance, if $X=Y=\frac{1}{2}, T$ fails to separate $M_{\frac{1}{2}}, \frac{1}{3}$ and $M_{\frac{1}{3}}, \frac{1}{2}$ as the second term compensates for the difference in the first. In their proof of full abstraction for probabilistic PCF, Ehrhard, Tasson and Pagani postpone the choice of X, Y, considering them instead as formal parameters. Valuations then become power series in these formal parameters.

Say we wish to extract from $M: A$ its valuation on $x \in \mathcal{E}_{\llbracket A \rrbracket}$. The valuation of $\llbracket \Downarrow_{x}^{A} M \rrbracket$ on $\{()\}$ is a power series, resulting from a sum over all successful interactions between $\llbracket M \rrbracket$ and $\llbracket \downarrow_{x}^{A} \rrbracket$ : one visiting exactly $x$, and possibly many others. But the one visiting $x$ is the only one visiting exactly once all components of the probabilistic sums in $\Downarrow_{x}^{A}$, i.e. the only one comprising all formal parameters exactly once. For instance, in our example above, the valuation of $M_{p, q}$ on $x$ is $\frac{q}{4}$, the coefficient of $\frac{q}{4} \mathrm{XY}+\frac{p}{4} \mathrm{Y}^{2}$ associated with the monomial where each parameter appears exactly once.

Let us now formalize this. Following [Ehrhard et al. 2014], if $P$ is a power series, then the $\mathcal{P}$ skeleton of $P$ is the coefficient of the monomial comprising each parameter of $\mathcal{P}$ exactly once. Then, we have, for any type $A$ and writing $\operatorname{FP}_{A}(x)$ for the set of parameters occurring in $\Downarrow_{x}^{A}$ :

Proposition 5.1. For any $x \in \mathcal{E}_{\llbracket A \rrbracket}, \mathrm{y} \in \mathcal{E}_{\llbracket}^{\cong} \overline{\underline{ }} \rrbracket$; the $\mathrm{FP}_{A}(x)$-skeleton of $\llbracket \Downarrow_{x}^{A} \rrbracket_{\mathrm{y}}$ is non-zero iff $x \in \mathrm{y}$.

From that and Equation 1, for $M, N: A$ and $\mathrm{x} \in \mathcal{E}_{\llbracket A \rrbracket}^{\cong}$ s.t. $\llbracket M \rrbracket_{\mathrm{x}} \neq \llbracket N \rrbracket_{\mathrm{x}}$, the $\mathrm{FP}_{A}(\underline{\mathrm{x}})$-skeleton of $\llbracket \downarrow_{\underline{\mathrm{x}}}^{A} M \rrbracket_{\{()\}}$is $\llbracket M \rrbracket_{\mathrm{x}} \times \alpha$ where $\alpha$ is the $\mathrm{FP}_{A}(\underline{\mathrm{x}})$-skeleton of $\llbracket \rrbracket_{\underline{\mathrm{x}}}^{A} \rrbracket$, and likewise for $\llbracket N \rrbracket_{\mathrm{x}}$. So $\llbracket \Downarrow_{\underline{\mathrm{x}}}^{A} M \rrbracket_{\mathrm{x}}$ and $\llbracket \downarrow_{\mathrm{x}}^{A} N \rrbracket_{\mathrm{x}}$ are power series differing in at least one coefficient. In the corresponding situation for probabilistic PCF, the authors of [Ehrhard et al. 2014] apply a result in analysis yielding $\rho \in[0,1]^{\mathrm{FP}_{A}(\underline{\mathrm{x}})}$ which separates them, hence $\Downarrow_{\underline{\mathrm{x}}}^{A}[\rho]$ a separating test.

5.1.2 Quantum Testing Terms. We now give testing and generation terms for quantum datatypes. For qbit, we should define a testing term $v: \mathbf{q b i t} \vdash \Downarrow_{\{\mathbf{q}\}}^{\mathbf{q b i t}}(v): 1$ and a generation term $\Uparrow_{\{\mathbf{q}\}}^{\mathbf{q b i t}}: \mathbf{q b i t}$.

Let us start by considering two terms $v:$ qbit $\vdash M, N: 1$ of the quantum $\lambda$-calculus. Their

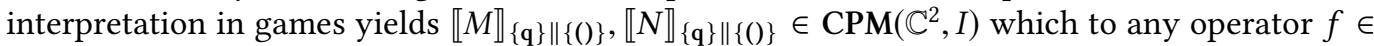
$\operatorname{Op}\left(\mathbb{C}^{2}\right)$ associates some $g \in \mathrm{Op}(\mathbb{C})$, i.e. a scalar factor in $\mathbb{C}$. But to test equality of maps in $\operatorname{CPM}\left(\mathbb{C}^{2}, I\right)$, it suffices to test them on hermitian operators, i.e. those $f \in \mathbf{O p}\left(\mathbb{C}^{2}\right)$ such that $f$ is equal to its conjugate transpose $f^{\dagger}$. Hermitians $\operatorname{Herm}\left(\mathbb{C}^{2}\right)$ on $\mathbb{C}^{2}$ form a 4-dimensional $\mathbb{R}$-vector space, admitting as basis the four positive Hermitian operators represented by the matrices

$$
\left(\begin{array}{ll}
1 & 0 \\
0 & 0
\end{array}\right) \quad\left(\begin{array}{ll}
0 & 0 \\
0 & 1
\end{array}\right) \quad \frac{1}{2}\left(\begin{array}{ll}
1 & 1 \\
1 & 1
\end{array}\right) \quad \frac{1}{2}\left(\begin{array}{cc}
1 & \mathbf{i} \\
-\mathbf{i} & 1
\end{array}\right) .
$$

We write $\mathbf{h}_{1}, \mathbf{h}_{2}, \mathbf{h}_{3}, \mathbf{h}_{4} \in \operatorname{CPM}\left(I, \mathbb{C}^{2}\right)$ for the corresponding completely positive maps. By construction, two $f, g \in \mathbf{C P M}\left(\mathbb{C}^{2}, I\right)$ are equal iff $f \circ \mathbf{h}_{i}=g \circ \mathbf{h}_{i}$ for $i \in\{1, \ldots, 4\}$. Besides, the $\mathbf{h}_{i}$ are definable, in the sense that there are $\vdash H_{1}, \ldots, H_{4}: \mathbf{q b i t}$ such that $\llbracket H_{i} \rrbracket_{\{\mathbf{q}\}}=\mathbf{h}_{i}$.

We must give one term $\Uparrow_{\{\mathbf{q}\}}^{\mathrm{qbit}}$ : qbit which will cover these four tests. We define it as

$$
\vdash \frac{1}{4} \mathrm{Z}_{1} \cdot H_{1}+\frac{1}{4} \mathrm{Z}_{2} \cdot H_{2}+\frac{1}{4} \mathrm{Z}_{3} \cdot H_{3}+\frac{1}{4} \mathrm{Z}_{4} \cdot H_{4}: \text { qbit }
$$


where $Z_{1}, \ldots, Z_{4}$ are fresh parameters. We write $\llbracket \Uparrow_{\{\mathbf{q}\}}^{\mathbf{q b i t}} \rrbracket_{\{\mathbf{q}\}}=\mathbf{h}_{\mathrm{Z}_{1}, \ldots, \mathrm{Z}_{4}} \in \mathbf{C P M}\left[\mathrm{Z}_{1}, \ldots, \mathrm{Z}_{4}\right]\left(I, \mathbb{C}^{2}\right)$, which through substitutions $\rho:\left\{Z_{1}, \ldots, Z_{4}\right\} \rightarrow\{0,1\}$ covers all the $\mathbf{h}_{i}$.

We must also define the testing term $\Downarrow_{\{\mathbf{q}\}}^{\mathbf{q b i t}}$. For that, we observe that for each $i \in\{1, \ldots, 4\}$, the dual $\mathbf{h}_{i}^{\dagger} \in \mathbf{C P M}\left(\mathbb{C}^{2}, I\right)$ of $\mathbf{h}_{i}$ (obtained via the dagger operation on CPM [Selinger 2007] - or equivalently, via the functorial action $(-)^{*}: \mathrm{CPM}^{\mathrm{op}} \rightarrow \mathrm{CPM}$ coming from the compact closure of CPM, followed by the canonical isomorphisms $\mathbb{C}^{2} \cong\left(\mathbb{C}^{2}\right)^{*}$ and $\left.\mathbb{C} \cong \mathbb{C}^{*}\right)$, may also be defined through terms $v: \mathbf{q b i t} \vdash H_{i}^{\dagger}(v): 1$ such that $\llbracket H_{i}^{\dagger} \rrbracket_{\{\mathbf{q}\} \|\{()\}}=\mathbf{h}_{i}^{\dagger}$; and we set $v: \mathbf{q b i t} \vdash \Downarrow_{\{\mathbf{q}\}}^{\mathbf{q b i t}}: 1$ as

$$
v: \text { qbit } \vdash \frac{1}{4} \mathrm{~V}_{1} \cdot H_{1}^{\dagger}(v)+\frac{1}{4} \mathrm{~V}_{2} \cdot H_{2}^{\dagger}(v)+\frac{1}{4} \mathrm{~V}_{3} \cdot H_{3}^{\dagger}(v)+\frac{1}{4} \mathrm{~V}_{4} \cdot H_{4}^{\dagger}(v): 1
$$

with fresh parameters $\mathrm{V}_{i}$. We write $\llbracket \Downarrow_{\{\mathbf{q}\} \|\{()\}}^{\mathrm{qbit}} \rrbracket_{\{\mathbf{q}\} \|\{()\}}=\mathbf{h}_{\mathrm{V}_{1}, \ldots, \mathrm{V}_{4}}^{\dagger} \in \mathbf{C P M}\left[\mathrm{V}_{1}, \ldots, \mathrm{V}_{4}\right]\left(\mathbb{C}^{2}, I\right)$.

This completes the definition of $\Downarrow_{x}^{A}$ and $\Uparrow_{x}^{A}$ for all types. We must now extend Proposition 5.1 for this completed definition, however this requires some disambiguation.

For $A$ a type, the parameters in $\Downarrow_{x}^{A}$ and $\pi_{x}^{A}$ may come from the classical clauses, or the quantum clauses. We reuse the notation $\mathrm{FP}_{A}(x)$ to denote the parameters arising from the classical clauses only, while $\mathrm{QP}_{A}(x)$ comprises those arising from quantum clauses. Now, if $A$ is a type and $x \in \mathcal{E}_{\llbracket A \rrbracket}$,

$$
\llbracket \Downarrow_{x}^{A} \rrbracket_{x \|\{()\}} \in \operatorname{CPM}\left[\operatorname{FP}_{A}(x) \uplus \mathrm{QP}_{A}(x)\right](\mathcal{H}(x), I)
$$

a polynomial with both kinds of formal parameters. We now consider its $\mathrm{FP}_{A}(x)$-skeleton to be the "coefficient" for the monomial comprising each parameter of $\mathrm{FP}_{A}(x)$ exactly once, i.e. the polynomial $P \in \mathrm{CPM}\left[\mathrm{QP}_{A}(x)\right](\mathcal{H}(x), I)$ such that $P \prod_{\mathrm{X} \in \mathrm{FP}_{A}(x)} \mathrm{X}$ is exactly the restriction of $\llbracket \Downarrow_{x}^{A} \rrbracket_{x} \|\{()\}$ to its monomials that comprise each parameter in $\mathrm{FP}_{A}(x)$ exactly once.

With this clarification, Proposition 5.1 holds for the full language, with exactly the same statement:

Proposition 5.2. For any $x \in \mathcal{E}_{\llbracket A \rrbracket}$, $\mathrm{y} \in \mathcal{E}_{\llbracket A \rrbracket}^{\cong}$; the $\mathrm{FP}_{A}(x)$-skeleton of $\llbracket \rrbracket_{x}^{A} \rrbracket_{y}$ is non-zero iff $x \in \mathrm{y}$.

The proof is by induction on $A$, following closely the intuition exposed in Section 5.1.1.

5.1.3 Quantum Properties. We now jump to the quantum properties of the test terms.

Lemma 5.3. Let $A$ be a type and $x \in \mathcal{E}_{\llbracket A \rrbracket}$. Then, the $\mathrm{FP}_{A}(x)$-skeleton of $\llbracket \Downarrow_{x}^{A} \rrbracket_{x} \|\{()\}$ is a polynomial

$$
P_{A, x} \in \mathrm{CPM}\left[\mathrm{QP}_{A}(x)\right](\mathcal{H}(x), I)
$$

and for all $f, g \in \operatorname{CPM}(I, \mathcal{H}(x)), f=g$ iff for all $\rho: \mathrm{QP}_{A}(x) \rightarrow\{0,1\}, P_{A, x}[\rho] \circ f=P_{A, x}[\rho] \circ g$.

To prove this, notice that for any $x \in \mathcal{E}_{\llbracket A \rrbracket}, \mathcal{H}(x)$ is, up to iso, some $\otimes_{1 \leq i \leq n} \mathbb{C}^{2}$ where $n$ is the number of qubits involved in $x$. Through this same isomorphism, $P_{A, x}$ relates to

$$
\otimes_{1 \leq i \leq n} \mathbf{h}_{\mathrm{V}_{1}^{i}, \ldots, \mathrm{V}_{4}^{i}}^{\dagger} \in \mathrm{CPM}\left[\mathrm{QP}_{A}(x)\right]\left(\otimes_{1 \leq i \leq n} \mathbb{C}^{2}, I\right)
$$

as follows by induction on $A$ and $x$. Now, the motivating property of $\mathbf{h}$ is stable under tensors - that is, the set $\otimes_{1 \leq i \leq n} \mathbf{h}_{\mathrm{V}_{1}^{i}, \ldots, \mathrm{V}_{4}^{i}}[\rho]$ for all $\rho: \mathrm{QP}_{A}(x) \rightarrow\{0,1\}$ covers a basis for all hermitian operators on $\otimes_{1 \leq i \leq n} \mathbb{C}^{2}$, as a $\mathbb{R}$-vector space. If $f, g \in \mathbf{C P M}\left(\otimes_{1 \leq i \leq n} \mathbb{C}^{2}, I\right)$ are equal on all of them, then they are equal. The dual property holds for $\otimes_{1 \leq i \leq n} \mathbf{h}_{\mathrm{V}_{1}^{i}, \ldots, \mathrm{V}_{4}^{i}}^{\dagger}$, from which the lemma follows.

\subsection{Full Abstraction}

We now prove our main result. If $\Gamma \vdash M, N: A$ are two homogeneously typed terms, we say they are observationally equivalent, written $M \equiv N$, iff for all context $C[-]$ such that $\vdash C[M], C[N]: 1$, the terms $C[M]$ and $C[N]$ have the same probability of convergence. 
THEOREM 5.4. The model $\mathrm{QA} / \equiv$ is fully abstract for the quantum $\lambda$-calculus, i.e. for all $\Gamma \vdash M, N: A$,

$$
M \equiv N \quad \Leftrightarrow \quad \llbracket M \rrbracket \equiv \llbracket N \rrbracket .
$$

Proof. In [Clairambault et al. 2019], the model is proved to be adequate with respect to an equivalence finer than $\equiv$, called simulation equivalence. As the two equivalences coincide on ground type, $\mathrm{QA} / \equiv$ is adequate, and if $\llbracket M \rrbracket \equiv \llbracket N \rrbracket$ it follows from standard arguments that $M \equiv N$.

For the converse, take $\Gamma \vdash M, N: A$ such that $\llbracket M \rrbracket \not \equiv \llbracket N \rrbracket$. For notational simplicity we consider $\Gamma$ empty. By hypothesis, there is $\mathrm{x} \in \mathcal{E}_{\bar{A}} \cong$ such that $\llbracket M \rrbracket_{\mathrm{x}} \neq \llbracket N \rrbracket_{\mathrm{x}}$. By Proposition 4.9, we have

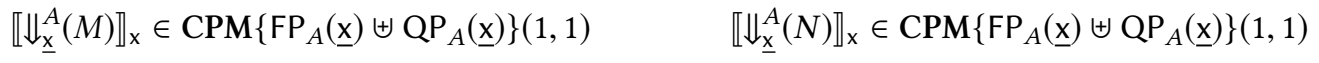

which are, in other words, power series with positive real coefficients, with domain of convergence

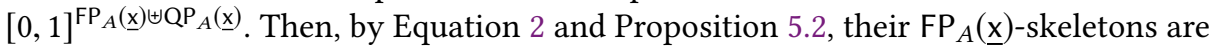

$$
P_{A, \underline{\mathrm{x}}} \circ \llbracket M \rrbracket_{\mathrm{x}} \in \mathrm{CPM}\left[\mathrm{QP}_{A}(\underline{\mathrm{x}})\right](1,1) \quad P_{A, \underline{\mathrm{x}}} \circ \llbracket N \rrbracket_{\mathrm{x}} \in \mathrm{CPM}\left[\mathrm{QP}{ }_{A}(\underline{\mathrm{x}})\right](1,1) .
$$

so by Lemma 5.3, since $\llbracket M \rrbracket_{\mathrm{x}} \neq \llbracket N \rrbracket_{\mathrm{x}}$ there must be $\mu: \mathrm{QP}_{A}(\underline{\mathrm{x}}) \rightarrow\{0,1\}$ such that $P_{A, \underline{\mathrm{x}}}[\mu] \circ \llbracket M \rrbracket_{\mathrm{x}} \neq$ $P_{A, \underline{x}}[\mu] \circ \llbracket N \rrbracket_{\mathrm{x}}$ are different positive reals. But then, we consider

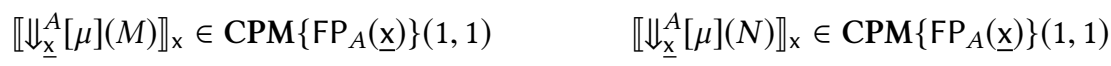

and in particular, their $\mathrm{FP}_{A}(\underline{\mathrm{x}})$-skeletons. Again by Equation 2 and Proposition 5.2, those must be respectively $P_{A, \underline{\mathrm{x}}}[\mu] \circ \llbracket M \rrbracket_{\mathrm{x}}$ and $P_{A, \underline{\mathrm{x}}}[\mu] \circ \llbracket N \rrbracket_{\mathrm{x}}$, which are known to be different! So $f=\llbracket \Downarrow_{\mathrm{x}}^{A}[\mu](M) \rrbracket_{\mathrm{x}}$ and $g=\llbracket \downarrow_{\mathrm{x}}^{A}[\mu](N) \rrbracket_{\mathrm{x}}$ are two power series with positive real coefficients, domain of convergence $[0,1]^{\mathrm{FP}_{A}(\underline{\mathrm{x}})}$, and at least one distinct coefficient. By Lemma 25 of [Ehrhard et al. 2014] applied to the substraction $f-g$, there is $\rho \in[0,1]^{\mathrm{FP}_{A}(\underline{\mathrm{x}})}$ such that $\llbracket \Downarrow_{\mathrm{x}}^{A}[\mu] M \rrbracket_{\mathrm{x}}[\rho] \neq \llbracket \Downarrow_{\mathrm{x}}^{A}[\mu] N \rrbracket_{\mathrm{x}}[\rho]$.

We finally form $T$ the term $v: A \vdash \Downarrow_{x}^{A}[\mu][\rho]: 1$. By the above and adequacy (Theorem 6.10 in [Clairambault et al. 2019]), $T(M)$ and $T(N)$ have a different probability of convergence.

\subsection{Collapse to Quantum Relations}

We first recall the quantum relational model [Pagani et al. 2014].

Definition 5.5. A quantum relational space (qrs) is $\mathfrak{A}=\left(d_{a}^{\mathfrak{A}}, G_{a}^{\mathfrak{A}}\right)_{a \in|\mathfrak{A}|}$ where $|\mathfrak{A}|$ is the web of $\mathfrak{A}$, for all $a \in|\mathfrak{A}|$ we have an integer $d_{a}^{\mathfrak{U}}$, and a sub-group $G_{a}^{\mathfrak{A}}$ of the group of permutations $\subseteq\left(d_{a}^{\mathfrak{A}}\right)$.

Intuitively, the web represents completed executions. If $A$ is a quantum game, the web of the corresponding $q r s$ is $\left|\int A\right|=\mathcal{E}_{\bar{A}}^{\cong}$ the set of symmetry classes of exhaustive configurations. For $\mathrm{x} \in\left|\int A\right|$, the dimension $d_{\mathrm{x}}$ is $\operatorname{simply} \operatorname{dim}\left(\mathcal{H}_{A}(\mathrm{x})\right)$. For the group of permutations $G_{\mathrm{x}}$, observe first that $\underline{\mathrm{x}} \cong_{A} \underline{\mathrm{x}}$ is a group of permutations on $\underline{\mathrm{x}}$. Identifying an integer $d$ with the set $\{0, \ldots, d-1\}$, any $\theta: \underline{\mathrm{x}} \cong_{A} \underline{\mathrm{x}}$ yields a bijection in $\mathfrak{S}\left(\Pi_{a \in \underline{\mathrm{x}}} \operatorname{dim}\left(\mathcal{H}_{A}(a)\right)\right)$ rearranging elements of the tuple following $\theta$, which in turn yields $\tilde{\theta} \in \mathfrak{S}\left(\operatorname{dim}\left(\mathcal{H}_{A}(\underline{\mathrm{x}})\right)\right)$, considering that $\operatorname{dim}\left(\mathcal{H}_{A}(\underline{\mathrm{x}})\right)=\operatorname{dim}\left(\bigotimes_{a \in \underline{\mathrm{x}}} \mathcal{H}_{A}(a)\right)=$ $\Pi_{a \in \mathrm{x}} \operatorname{dim}\left(\mathcal{H}_{A}(a)\right)$ and following the bijection induced by the lexicographic ordering.

5.3.1 Constructions on qrs and Compatibility with Games. We now introduce some constructions on qrs, overall defining an interpretation $(A)$ as a qrs of all types $A$ of the quantum $\lambda$-calculus. We first set $(1)=(1,\{\mathrm{id}\})_{a \in\{*\}}$ and $(\mathbf{q b i t})=(2,\{\mathrm{id}\})_{a \in\{*\}}$. If $\mathfrak{A}$ and $\mathfrak{B}$ are qrs, then $\mathfrak{A} *=\mathfrak{A}$; and $\mathfrak{A} \otimes \mathfrak{B}$ is defined as $|\mathfrak{A} \otimes \mathfrak{B}|=|\mathfrak{A}| \times|\mathfrak{B}|, d_{(a, b)}=d_{a} \cdot d_{b}$. For $G_{(a, b)}$, consider first the set of permutations on $d_{a} \cdot d_{b}$ whose action is induced by $f \in G_{a}, g \in G_{b}$ via $h(i, j)=(f(i), g(j))$ - this set induces a group of permutations $G_{(a, b)}$ on $d_{(a, b)}$ again through the lexicographic ordering.

For the exponential (!), we need some notations and terminology on multisets. If $A$ is a set, let $\mathscr{M}(A)$ denote multisets on $A$, defined as functions $\mu: A \rightarrow \mathbb{N}$ indicating, for each element $a \in A$, its multiplicity $\mu(a)$. We write $v(\mu)$ for its support, i.e. the set of $a \in|\mathfrak{A}|$ such that $\mu(a) \neq 0$. We say 
that $\mu$ is finite if it has finite support. In that case, its cardinality $\sum_{a \in A} \mu(a)$ is also finite. We write $\mathscr{M}_{f}(A)$ for the set of finite multisets on $A$, and $\mathscr{M}_{k}(A)$ for the multisets of cardinality $k$.

For $\mathfrak{A}$ a qrs, the qrs ! $\mathfrak{A}$ is constructed in two steps. First, we build the symmetric tensor product $\mathfrak{A}^{\odot k}$ representing $k$ unordered uses of the resource $\mathfrak{A}$. Its web is $\left|\mathfrak{H}{ }^{\odot k}\right|=\mathscr{M}_{k}(|\mathfrak{A}|)$. For $\mu \in \mathscr{M}_{k}(|\mathfrak{A}|)$, the dimension is $d_{\mu}=\prod_{a \in v(\mu)} d_{a}^{\mu(a)}$. The group $G_{\mu}$ comprises those permutations on $\prod_{a \in v(\mu)} d_{a}^{\mu(a)}$ given by, for all $a \in v(\mu)$, a permutation $g_{a} \in \mathfrak{S}\left(d_{a}^{\mu(a)}\right)$ acting as

$$
g_{a}\left(i_{0}, \ldots, i_{\mu(a)-1}\right)=\left(g_{a}^{0}\left(i_{\pi(0)}\right), \ldots, g_{n}^{\mu(a)-1}\left(i_{\pi(\mu(a)-1)}\right)\right)
$$

following some $\pi \in \mathfrak{S}(\mu(a))$ between copies, and, for each $0 \leq i \leq \mu(a)-1$, some $g_{a}^{i} \in G_{a}$.

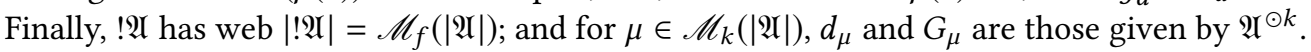

We omit the qrs construction for sums (and lists), which may be found in [Pagani et al. 2014]. Altogether, these constructions define an interpretation of types of the quantum $\lambda$-calculus as qrs, with all cases transparent except for $(A \multimap B)=(A)^{*} \otimes(B)$ and $(\mid !(A \multimap B))=!\left((\mid A)^{*} \otimes(B)\right)$.

To compare these with the corresponding arena constructions, we introduce a strong equivalence between qrs. A renaming from qrs $\mathfrak{A}$ to $\mathfrak{B}$ is a pair $\alpha=\left(\alpha^{1},\left(\alpha_{a}^{2}\right)_{a \in|\mathfrak{X}|}\right)$ comprising $\alpha^{1}:|\mathfrak{A}| \rightarrow|\mathfrak{B}|$ a bijection, and for each $a \in|\mathfrak{A}|$, a bijection $\alpha_{a}^{2}: d_{a}^{\mathfrak{I}} \rightarrow d_{\alpha^{1}(a)}^{\mathfrak{B}}$, transporting $G_{a}^{\mathfrak{A}}$ to $G_{\alpha^{1}(a)}^{\mathfrak{B}}$ by conjugacy.

Proposition 5.6. For any arenas $A, B$, we have renamings

$$
\begin{aligned}
& r^{1}: \quad(1) \cong \int \llbracket 1 \rrbracket \quad r_{A, B}^{\oplus}:\left(\int A\right) \oplus\left(\int B\right) \cong \int(A \oplus B) \\
& r^{\text {qbit }}: \quad(\mathbf{q b i t}) \cong \int \llbracket \mathbf{q b i t} \rrbracket \quad r_{A, B}^{-0}:\left(\int A\right)^{*} \otimes\left(\int B\right) \cong \quad \int(A \multimap B) \\
& r_{A, B}^{\otimes}:\left(\int A\right) \otimes\left(\int B\right) \cong \int(A \otimes B) \quad r_{A, B}^{!}: \quad !\left(\int(A \multimap B)\right) \cong \int(!(A \multimap B))
\end{aligned}
$$

yielding, overall, a renaming $r^{A}:(A) \cong \int \llbracket A \rrbracket$ for every type $A$ of the quantum $\lambda$-calculus.

Proof. Direct verification, also using that $\otimes, \oplus, !$ act functorially on renamings. These renamings extend smoothly to $n$-ary tensors and countable sums, covering the list constructor as well.

5.3.2 Morphisms of qrs. Now, we consider what are the morphisms between qrs, forming a category QRS. Ignoring symmetry at first, the intension is to set simply morphisms in QRS( $\mathfrak{A}, \mathfrak{B})$ to be matrices $\left(\alpha_{a, b}\right)_{(a, b) \in|\mathfrak{I}| \times|\mathfrak{B}|}$ such that, for all $(a, b) \in|\mathfrak{A}| \times|\mathfrak{B}|, \alpha_{a, b} \in \mathrm{CPM}\left(\mathbb{C}^{d_{a}^{A}}, \mathbb{C}^{d_{b}^{B}}\right)$.

However, these coefficients must also be invariant under symmetry. To express that, note that each $g \in G_{a}^{\mathfrak{I}}$ induces $\widehat{g} \in \operatorname{CPM}\left(\mathbb{C}^{d_{a}^{A}}, \mathbb{C}^{d_{a}^{A}}\right)$ in the obvious way, and just as in Definition 4.2, we set

$$
\gamma_{\mathfrak{R}, a}=\frac{1}{\left|G_{a}^{\mathfrak{N}}\right|} \sum_{g \in G_{a}^{\mathfrak{N}}} \widehat{g} .
$$

where $\left|G_{a}^{\mathfrak{A}}\right|$ denotes the cardinal of the group - hopefully the overload of $|-|$ creates no confusion. Invariance of $\alpha$ under symmetry is then stated as $\gamma_{\mathfrak{A}, a} \circ \alpha_{a, b} \circ \gamma_{\mathfrak{B}, b}=\alpha_{a, b}$ for all $(a, b) \in|\mathfrak{U}| \times|\mathfrak{B}|$.

This does not yet conclude the construction of QRS: an issue arises with composition. Consider $\left(\alpha_{a, b}\right)_{(a, b) \in|\mathfrak{A}| \times|\mathfrak{B}|}$ and $\left(\beta_{b, c}\right)_{(b, c) \in|\mathfrak{B}| \times|\mathfrak{C}|}$ invariant under symmetry. Their composition is to be

$$
(\beta \odot \alpha)_{a, c}=\sum_{b \in|\mathfrak{B}|} \beta_{b, c} \circ \alpha_{a, b},
$$

however this sum is in general infinite, and there is no reason why it would always converge. Therefore, QRS $(\mathfrak{A}, \mathfrak{B})$ is the D-completion of the set of those $f \in \operatorname{CPM}\left(\mathbb{C}^{d_{a}^{A}}, \mathbb{C} d_{b}^{B}\right)$ invariant under symmetry, partially ordered by the Löwner order. Altogether, by this construction we obtain a category QRS. Morphisms are composed via Equation 5.3.2, where the sum is known to converge thanks to D-completion. Identity on $\mathfrak{A}$ has id $\operatorname{la}_{a, a^{\prime}}$ set to 0 if $a \neq a^{\prime}$, and $\gamma_{\mathfrak{I}, a}$ otherwise. It is proved in [Pagani et al. 2014] that QRS forms a compact closed category, with biproducts given by $\oplus$, 
and furthermore that for any $\mathfrak{A}, ! \mathfrak{A}$ is a free commutative comonoid, altogether forming a Lafont category [Melliès 2009]. Relying on this along with standard interpretations of quantum primitives in CPM, there is an adequate interpretation of terms $\Gamma \vdash M: A$ as morphisms $(M) \in \mathrm{QRS}((\Gamma), \mid(A))$.

5.3.3 From QA to QRS. The construction $\int(-)$ extends to a functor $\int(-): \mathrm{QA} \rightarrow \mathrm{QRS}$ defined on objects as above. On $\sigma: S \rightarrow A^{\perp \not 8} B$, we define $\int \sigma \in \mathrm{QRS}\left(\int A, \int B\right) \operatorname{simply}$ via $\left(\int \sigma\right)_{\mathrm{x}_{A}, \mathrm{x}_{B}}=\sigma_{\mathrm{x}_{A}, \mathrm{x}_{B}}$ as in Section 4.1. Functoriality is exactly Equation 1 established in Section 4.2. Observe that $\equiv$ is exactly the equivalence relation on $\mathrm{QA}(A, B)$ induced by $\int(-): \sigma \equiv \sigma^{\prime}$ iff $\int \sigma=\int \sigma^{\prime}$.

Furthermore, $\int(-)$ preserves all the structure used in the interpretation. The interpretations in QA and QRS are phrased in slightly different ways. In [Pagani et al. 2014], QRS is shown to be compact closed with biproducts and a Lafont exponential. In contrast, being more intensional, QA has the more elaborate structure described in Section 3.4.2, that we may call a linear closed Freyd category with coproducts along with a linear exponential comonad acting on a sub-smc including the linear arrow types. Those differences are superficial: QRS also forms a linear closed Freyd category with $\mathrm{QRS}^{t}=\mathrm{QRS}$ with the adjunction given by duality of the compact closed structure; and every Lafont category yields a linear exponential comonad on the linear category [Bierman 1993].

THEOREM 5.7. There is a strong monoidal functor $\int(-):$ QA $\rightarrow$ QRS preserving all categorical components used in the interpretation up to coherent isomorphism. It follows that for any $\vdash M: A$, $\int \llbracket M \rrbracket=r^{A} \circ(M)$ (where $r^{A}$ is an iso lifted from the renaming of Proposition 5.6).

Proof. Preservation of identity is idempotence of $\gamma_{\underline{x}_{A}, \underline{x}_{A}}^{A}$. The renamings of Proposition 5.6 are lifted to isomorphisms in QRS: for instance, $\alpha=\left(\alpha^{1},\left(\alpha_{a}^{2}\right)_{a \in|\mathfrak{M}|}\right)$ from $\mathfrak{A}$ to $\mathfrak{B}$ yields $\alpha_{a, b}=0$ if $b \neq \alpha^{1}(a)$, and $\alpha_{a, b}=\widehat{\alpha_{a}^{2}} \in \operatorname{CPM}\left(\mathbb{C}^{d_{a}^{\mathfrak{9}}}, \mathbb{C}_{b}^{d_{b}^{\mathfrak{B}}}\right)$ otherwise. Those are natural and verify the expected coherence conditions. Preservation of the rest of the structure follows similar lines. From this and direct verification for the interpretation of primitives of the quantum $\lambda$-calculus, the compatibility of the collapse $\int(-)$ with the interpretation also follows.

Although the construction of QRS requires D-completion, the collapse $\int(-)$ only reaches finite elements (note that in [Pagani et al. 2014] it was already proved that the interpretation of the quantum $\lambda$-calculus in QRS only reaches finite elements). We deduce our final result:

THEOREM 5.8. The interpretation of the quantum $\lambda$-calculus in QRS is fully abstract.

Proof. Since QRS is adequate, only one direction remains. Let $\vdash M, N: A$ be such that $M \equiv N$. Then, $\llbracket M \rrbracket \equiv \llbracket N \rrbracket$ by Theorem 5.4. So, $\int \llbracket M \rrbracket=\int \llbracket N \rrbracket$, thus $(M)=(N)$ by Theorem 5.7.

\section{ACKNOWLEDGMENTS}

We are grateful to the anonymous reviewers for their helpful comments and suggestions.

This work is supported by ANR project DyVerSe (ANR-19-CE48-0010-01) and Labex MiLyon (ANR-10-LABX-0070) of Université de Lyon, within the program "Investissements d'Avenir" (ANR11-IDEX-0007), operated by the French National Research Agency (ANR).

\section{REFERENCES}

Samson Abramsky, Radha Jagadeesan, and Pasquale Malacaria. 2000. Full Abstraction for PCF. Inf. Comput. 163, 2 (2000), 409-470. https://doi.org/10.1006/inco.2000.2930

Samson Abramsky and Guy McCusker. 1996. Linearity, Sharing and State: a fully abstract game semantics for Idealized Algol with active expressions. Electr. Notes Theor. Comput. Sci. 3 (1996), 2-14. https://doi.org/10.1016/S1571-0661(05)80398-6

Samson Abramsky and Paul-André Melliès. 1999. Concurrent Games and Full Completeness. In 14th Annual IEEE Symposium on Logic in Computer Science, Trento, Italy, Fuly 2-5, 1999. 431-442. https://doi.org/10.1109/LICS.1999.782638 
Patrick Baillot, Vincent Danos, Thomas Ehrhard, and Laurent Regnier. 1997. Timeless Games. In Computer Science Logic, 11th International Workshop, CSL '97, Annual Conference of the EACSL, Aarhus, Denmark, August 23-29, 1997, Selected Papers. 56-77. https://doi.org/10.1007/BFb0028007

Gavin Bierman. 1993. On intuitionistic linear logic. Ph.D. Dissertation. University of Cambridge Computer Laboratory.

Pierre Boudes. 2009. Thick Subtrees, Games and Experiments. In Typed Lambda Calculi and Applications, 9th International Conference, TLCA 2009, Brasilia, Brazil, July 1-3, 2009. Proceedings. 65-79. https://doi.org/10.1007/978-3-642-02273-9_7

Ana C. Calderon and Guy McCusker. 2010. Understanding Game Semantics Through Coherence Spaces. Electr. Notes Theor. Comput. Sci. 265 (2010), 231-244. https://doi.org/10.1016/j.entcs.2010.08.014

Simon Castellan. 2017. Concurrent structures in game semantics. (Structures concurrentes en sémantique des jeux). Ph.D. Dissertation. University of Lyon, France. https:/tel.archives-ouvertes.fr/tel-01587718

Simon Castellan and Pierre Clairambault. 2016. Causality vs. Interleavings in Concurrent Game Semantics. In 27th International Conference on Concurrency Theory, CONCUR 2016, August 23-26, 2016, Québec City, Canada. 32:1-32:14. https://doi.org/10.4230/LIPIcs.CONCUR.2016.32

Simon Castellan, Pierre Clairambault, Hugo Paquet, and Glynn Winskel. 2018. The concurrent game semantics of Probabilistic PCF. In Proceedings of the 33rd Annual ACM/IEEE Symposium on Logic in Computer Science, LICS 2018, Oxford, UK, fuly 09-12, 2018. 215-224. https://doi.org/10.1145/3209108.3209187

Simon Castellan, Pierre Clairambault, Silvain Rideau, and Glynn Winskel. 2017. Games and Strategies as Event Structures. LMCS 13, 3 (2017).

Simon Castellan, Pierre Clairambault, and Glynn Winskel. 2014. Symmetry in concurrent games. In foint Meeting of the Twenty-Third EACSL Annual Conference on Computer Science Logic (CSL) and the Twenty-Ninth Annual ACM/IEEE Symposium on Logic in Computer Science (LICS), CSL-LICS '14, Vienna, Austria, fuly 14 - 18, 2014. 28:1-28:10. https: //doi.org/10.1145/2603088.2603141

Simon Castellan, Pierre Clairambault, and Glynn Winskel. 2015. The Parallel Intensionally Fully Abstract Games Model of PCF. In 30th Annual ACM/IEEE Symposium on Logic in Computer Science, LICS 2015, Kyoto, Japan, July 6-10, 2015. 232-243. https://doi.org/10.1109/LICS.2015.31

Simon Castellan, Pierre Clairambault, and Glynn Winskel. 2019. Thin Games with Symmetry and Concurrent Hyland-Ong Games. Logical Methods in Computer Science 15, 1 (2019). https://mcs.episciences.org/5248

Simon Castellan and Nobuko Yoshida. 2019. Two sides of the same coin: session types and game semantics: a synchronous side and an asynchronous side. PACMPL 3, POPL (2019), 27:1-27:29. https://doi.org/10.1145/3290340

Pierre Clairambault, Marc de Visme, and Glynn Winskel. 2019. Game semantics for quantum programming. PACMPL 3 , POPL (2019), 32:1-32:29. https://doi.org/10.1145/3290345

Pierre Clairambault, Julian Gutierrez, and Glynn Winskel. 2012. The Winning Ways of Concurrent Games. In Proceedings of the 27th Annual IEEE Symposium on Logic in Computer Science, LICS 2012, Dubrovnik, Croatia, fune 25-28, 2012. 235-244. https://doi.org/10.1109/LICS.2012.34

J.R.B. Cockett and R.A.G. Seely. 1997. Weakly distributive categories. Journal of Pure and Applied Algebra 114, 2 (1997), 133-173.

Ugo Dal Lago and Olivier Laurent. 2008. Quantitative Game Semantics for Linear Logic. In Computer Science Logic, 22nd International Workshop, CSL 2008, 17th Annual Conference of the EACSL, Bertinoro, Italy, September 16-19, 2008. Proceedings. 230-245. https://doi.org/10.1007/978-3-540-87531-4_18

Yannick Delbecque. 2011. Game Semantics for Quantum Data. Electr. Notes Theor. Comput. Sci. 270, 1 (2011), 41-57. https://doi.org/10.1016/j.entcs.2011.01.005

Peter Dybjer and Andrzej Filinski. 2000. Normalization and Partial Evaluation. In Applied Semantics, International Summer School, APPSEM 2000, Caminha, Portugal, September 9-15, 2000, Advanced Lectures. 137-192.

Thomas Ehrhard. 2012. The Scott model of linear logic is the extensional collapse of its relational model. Theor. Comput. Sci. 424 (2012), 20-45. https://doi.org/10.1016/j.tcs.2011.11.027

Thomas Ehrhard, Christine Tasson, and Michele Pagani. 2014. Probabilistic coherence spaces are fully abstract for probabilistic PCF. In The 41st Annual ACM SIGPLAN-SIGACT Symposium on Principles of Programming Languages, POPL '14, San Diego, CA, USA, January 20-21, 2014. 309-320. https://doi.org/10.1145/2535838.2535865

Nicolas Gisin, Grégoire Ribordy, Wolfgang Tittel, and Hugo Zbinden. 2002. Quantum cryptography. Reviews of modern physics 74, 1 (2002), 145.

Lov K. Grover. 1996. A Fast Quantum Mechanical Algorithm for Database Search. In Proceedings of the Twenty-Eighth Annual ACM Symposium on the Theory of Computing, Philadelphia, Pennsylvania, USA, May 22-24, 1996. 212-219.

Russell Harmer and Guy McCusker. 1999. A Fully Abstract Game Semantics for Finite Nondeterminism. In 14th Annual IEEE Symposium on Logic in Computer Science, Trento, Italy, fuly 2-5, 1999. 422-430. https://doi.org/10.1109/LICS.1999.782637

Ichiro Hasuo and Naohiko Hoshino. 2017. Semantics of higher-order quantum computation via geometry of interaction. Ann. Pure Appl. Logic 168, 2 (2017), 404-469. https://doi.org/10.1016/j.apal.2016.10.010 
J. M. E. Hyland and C.-H. Luke Ong. 2000. On Full Abstraction for PCF: I, II, and III. Inf. Comput. 163, 2 (2000), $285-408$. https://doi.org/10.1006/inco.2000.2917

Martin Hyland and Andrea Schalk. 1999. Abstract Games for Linear Logic. Electr. Notes Theor. Comput. Sci. 29 (1999), 127-150. https://doi.org/10.1016/S1571-0661(05)80312-3

Martin Hyland and Andrea Schalk. 2003. Glueing and orthogonality for models of linear logic. Theor. Comput. Sci. 294, 1/2 (2003), 183-231. https://doi.org/10.1016/S0304-3975(01)00241-9

André Joyal, Ross Street, and Dominic Verity. 1996. Traced monoidal categories. In Mathematical Proceedings of the Cambridge Philosophical Society, Vol. 119. Cambridge University Press, 447-468.

Jim Laird, Giulio Manzonetto, and Guy McCusker. 2013. Constructing differential categories and deconstructing categories of games. Inf. Comput. 222 (2013), 247-264. https://doi.org/10.1016/j.ic.2012.10.015

Octavio Malherbe. 2013. Categorical models of computation: partially traced categories and presheaf models of quantum computation. Ph.D. Dissertation. University of Ottawa.

Octavio Malherbe, Philip Scott, and Peter Selinger. 2013. Presheaf Models of Quantum Computation: An Outline. In Computation, Logic, Games, and Quantum Foundations. The Many Facets of Samson Abramsky - Essays Dedicated to Samson Abramsky on the Occasion of His 60th Birthday. 178-194. https://doi.org/10.1007/978-3-642-38164-5_13

Paul-André Melliès. 2005. Asynchronous Games 4: A Fully Complete Model of Propositional Linear Logic. In 20th IEEE Symposium on Logic in Computer Science (LICS 2005), 26-29 June 2005, Chicago, IL, USA, Proceedings. 386-395. https: //doi.org/10.1109/LICS.2005.6

Paul-André Melliès. 2006. Asynchronous games 2: The true concurrency of innocence. Theor. Comput. Sci. 358, 2-3 (2006), 200-228. https://doi.org/10.1016/j.tcs.2006.01.016

Paul-André Melliès and Nicolas Tabareau. 2010. Resource modalities in tensor logic. Ann. Pure Appl. Logic 161, 5 (2010), 632-653. https://doi.org/10.1016/j.apal.2009.07.018

Paul-André Melliès. 2009. Categorical semantics of linear logic. Panoramas et syntheses 27 (2009), 15-215

Robin Milner. 1977. Fully Abstract Models of Typed lambda-Calculi. Theor. Comput. Sci. 4, 1 (1977), 1-22.

Andrzej S. Murawski and C.-H. Luke Ong. 2003. Exhausting strategies, joker games and full completeness for IMLL with Unit. Theor. Comput. Sci. 294, 1/2 (2003), 269-305. https://doi.org/10.1016/S0304-3975(01)00244-4

Michele Pagani, Peter Selinger, and Benoît Valiron. 2014. Applying quantitative semantics to higher-order quantum computing. In The 41st Annual ACM SIGPLAN-SIGACT Symposium on Principles of Programming Languages, POPL '14, San Diego, CA, USA, January 20-21, 2014. 647-658. https://doi.org/10.1145/2535838.2535879

John Power and Edmund Robinson. 1997. Premonoidal Categories and Notions of Computation. Mathematical Structures in Computer Science 7, 5 (1997), 453-468. https://doi.org/10.1017/S0960129597002375

John Power and Hayo Thielecke. 1999. Closed Freyd- and kappa-categories. In ICALP'99 (LNCS), Vol. 1644. Springer.

Silvain Rideau and Glynn Winskel. 2011. Concurrent Strategies. In LICS '11, fune 21-24, 2011, Toronto, Canada. 409-418.

Peter Selinger. 2004. Towards a quantum programming language. Mathematical Structures in Computer Science 14, 4 (2004), 527-586. https://doi.org/10.1017/S0960129504004256

Peter Selinger. 2007. Dagger Compact Closed Categories and Completely Positive Maps: (Extended Abstract). Electr. Notes Theor. Comput. Sci. 170 (2007), 139-163. https://doi.org/10.1016/j.entcs.2006.12.018

Peter Selinger and Benoît Valiron. 2006. A lambda calculus for quantum computation with classical control. Mathematical Structures in Computer Science 16, 3 (2006), 527-552. https://doi.org/10.1017/S0960129506005238

Peter Selinger and Benoît Valiron. 2008. On a Fully Abstract Model for a Quantum Linear Functional Language: (Extended Abstract). Electr. Notes Theor. Comput. Sci. 210 (2008), 123-137. https://doi.org/10.1016/j.entcs.2008.04.022

Peter W. Shor. 1997. Polynomial-Time Algorithms for Prime Factorization and Discrete Logarithms on a Quantum Computer. SIAM f. Comput. 26, 5 (1997), 1484-1509. https://doi.org/10.1137/S0097539795293172

Takeshi Tsukada and C.-H. Luke Ong. 2015. Nondeterminism in Game Semantics via Sheaves. In 30th Annual ACM/IEEE Symposium on Logic in Computer Science, LICS 2015, Kyoto, Japan, fuly 6-10, 2015. 220-231.

Glynn Winskel. 2007. Event Structures with Symmetry. Electr. Notes Theor. Comput. Sci. 172 (2007), 611-652. https: //doi.org/10.1016/j.entcs.2007.02.022

Glynn Winskel. 2015. On Probabilistic Distributed Strategies. In Theoretical Aspects of Computing - ICTAC 2015 - 12 th International Colloquium Cali, Colombia, October 29-31, 2015, Proceedings. 69-88.

Dongsheng Zhao and Taihe Fan. 2010. Dcpo-completion of posets. Theor. Comput. Sci. 411, 22-24 (2010), $2167-2173$. https://doi.org/10.1016/j.tcs.2010.02.020 